\title{
COMPLETIONS IN ABSTRACT HOMOTOPY THEORY
}

\author{
BY \\ ALEX HELLER( ${ }^{(1)}$
}

Introduction. Certain formal theorems of homotopy theory occur in a wide variety of contexts in algebra as well as in topology. The notion of an h-c-category was introduced in [6] in order to provide a common framework for their several occurrences.

In this paper the considerations of [6] will be supplemented by a theory of completions of h-c-categories. The utility of such a theory is most strikingly demonstrated by the remarkable representation theorem for half-exact functors due to E. H. Brown [2]. It may also play a role in derivations of the spectral sequences of Adams for stable homotopy theory and of Dyer-Kahn for fibre bundles. These matters, however, are not within the scope of this paper.

The primary example, which provides the intuitive basis for the notion of an $\mathrm{h}$-c-category, is that of the category of finite $\mathrm{CW}$-complexes. The completion in this case is the category of all $\mathrm{CW}$-complexes. The peculiarities of the relevant notion of completion are quite evident in this case: the latter category is neither complete nor cocomplete in the usual senses of category theory. What is the case is that certain families, e.g., increasing families of subcomplexes, have colimits.

Starting with this model Boardman [1] defined a completion of the stabilized category of finite $\mathrm{CW}$-complexes which seems to be generally recognized as the appropriate category for stable homotopy theory. The concern of this paper is at once to generalize and to amplify the work of Boardman. The generalization lies in the fact that the constructions are applicable to all h-c-categories, rather than just to the category of finite h-c-complexes. The amplification is the observation that their application leads to new h-c-categories to which the formal theorems of homotopy theory are still applicable.

An h-c-category $(\$ 1)$ is a category with two additional items of structure: a family of morphisms called cofibrations, which makes it a c-category, and a congruence called homotopy which, together with the family of cofibrations, makes it an h-c-category. For c-completeness of a c-category one requires the existence of colimits of a certain very special class of functors called c-systems ( $\$ 3)$, indexed by lattices. For a fixed index lattice the c-systems themselves form a c-category and the colimit functor is to preserve the appropriate structure. The c-completion of a small c-category always exists, and is characterized by a universal property ( $\$ 6-8)$.

Received by the editors November 7, 1968.

( ${ }^{1}$ ) This research was in part supported by NSF grant GP 7964.

Copyright (C) 1970, American Mathematical Society 
If this small category is an h-c-category then the categories of c-systems as well as the c-completion are also h-c-categories ( $\$ 9-10)$.

The homotopy category of a c-completion is closed under coproducts and products and has the stronger completeness property that any contravariant functor satisfying the obvious necessary conditions for representability is in fact representable ( $\$ 12$, this is Brown's theorem).

If the original category is provided with a suitable "smash product" ( $(14)$, then this product extends to the completion. Under these circumstances the homotopy category becomes a monoidal closed category (cf. [4] for this notion).

When an h-c-category possesses a suspension functor $(\$ 13)$ it may be stabilized with respect to this functor, i.e., the suspension may be made into an automorphism. The resulting category is again an h-c-category. This process does not commute with completion; it is Boardman's observation that in order to get a complete stable homotopy category the appropriate order is to stabilize first and then complete.

The behavior of smash products under stabilization is an interesting matter which is not completely elucidated here. There remain some unsolved technical problems and the treatment given below $(\$ 15)$ must be regarded as a preliminary sketch.

1. h-c-categories. We begin by recalling (cf. [6]) the axioms for an h-c-category.

A c-category is a pair $(\mathscr{C}, \operatorname{Cof} \mathscr{C})$ where $\mathscr{C}$ is a pointed category with initial and terminal object $O$ and $\operatorname{Cof} \mathscr{C}$ is a subcategory $\left({ }^{2}\right)$ such that

(C1) All isomorphisms and all morphisms $O \rightarrow A$ are in Cof $\mathscr{C}$.

(C2) If $a: A^{\prime} \rightarrow A$ is in Cof $\mathscr{C}$ then for any $f^{\prime}: A^{\prime} \rightarrow B$ the pushout

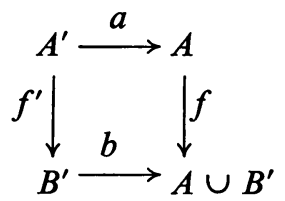

exists in $\mathscr{C}$ and $b \in \operatorname{Cof} \mathscr{C}$.

The morphisms in Cof $\mathscr{C}$ are called cofibrations; diagrams such as 1.1 are referred to as c-pushouts.

It follows that cofibrations have cokernels, called cofibres, and that finite coproducts, which we denote with the symbol $\vee$, exist in $\mathscr{C}$.

A functor from one c-category to another is a c-functor if it preserves cofibrations and c-pushouts. A c-subcategory of a c-category $(\mathscr{C}$, Cof $\mathscr{C})$ is a full subcategory $\mathscr{C}^{\prime}$ which is itself a c-category when provided with $\operatorname{Cof} \mathscr{C}^{\prime}=\mathscr{C}^{\prime} \cap \operatorname{Cof} \mathscr{C}$ as cofibrations, and is further closed under c-pushouts in $\mathscr{C}$.

An h-c-category is a c-category $(\mathscr{C}$, Cof $\mathscr{C})$ provided with a congruence $\simeq$, called homotopy, satisfying the following axioms.

(2) We depart slightly from the notation of [6], where Cof $\mathscr{C}$ stood for the full subcategory of the morphism category whose objects are discussed here. 
(HC1) (additivity). If $f^{\prime} \simeq g^{\prime}: A^{\prime} \rightarrow B$ and $f^{\prime \prime} \simeq g^{\prime \prime}: A^{\prime \prime} \rightarrow B$ then $\left(f^{\prime} f^{\prime \prime}\right) \simeq\left(g^{\prime} g^{\prime \prime}\right)$ : $A^{\prime} \vee A^{\prime \prime} \rightarrow B$.

(HC2) (homotopy extension). If

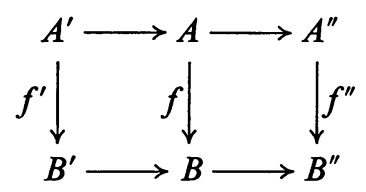

commutes, the rows being cofibrations followed by their cofibres, and $g^{\prime} \simeq f^{\prime}$ then there are $g \simeq f, g^{\prime \prime} \simeq f^{\prime \prime}$ such that the diagram obtained by substituting the $g$ 's for the $f$ 's again commutes.

(HC3) (deformation-retraction). If in (1.1) $a$ is a homotopy equivalence, i.e., if its image in $\mathscr{C} \square=\mathscr{C} / \simeq$ is an isomorphism, then so is $b$.

(HC4) (mapping cylinder). Any morphism in $\mathscr{C}$ may be factored in the form $g f$ with $f$ a cofibration and $g$ a homotopy equivalence.

An h-c-functor is a c-functor which preserves the homotopy relation.

In [6], we showed that the elementary operations of homotopy theory may be carried out in any h-c-category; inter alia the suspension $\Sigma: \mathscr{C} \square \rightarrow \mathscr{C} \square$ and the Puppe sequence of a cofibration are always defined. We shall use the results of [6] freely below; in addition, we shall need the following two lemmas of elementary homotopy theory.

LEMMA 1.2. If, in the diagram $1.1, f^{\prime}$ is a homotopy equivalence, then so is $f$.

We recall that a cylinder over $A^{\prime}$ is a mapping cylinder

$$
A^{\prime} \vee A^{\prime} \stackrel{\left(i_{0}^{\prime} i_{1}^{\prime}\right)}{\longrightarrow} Z A^{\prime} \longrightarrow A
$$

for the folding morphism (1 1 ): $A^{\prime} \vee A^{\prime} \rightarrow A^{\prime}$. We may construct a cylinder over $A$ in such a way that $\left(i_{0} z i_{1}\right): A \cup_{0} Z A^{\prime} \cup_{1} A \rightarrow Z A$ is a cofibration, where $A \cup_{0} Z A^{\prime}$ $\cup_{1} A$ is the pushout of $\left(i_{0}^{\prime} i_{1}^{\prime}\right)$ and $a$; the notation $A \cup_{0} Z A^{\prime} \cup_{1} A$ is intended to be mnemonic.

We now proceed to construct the diagram

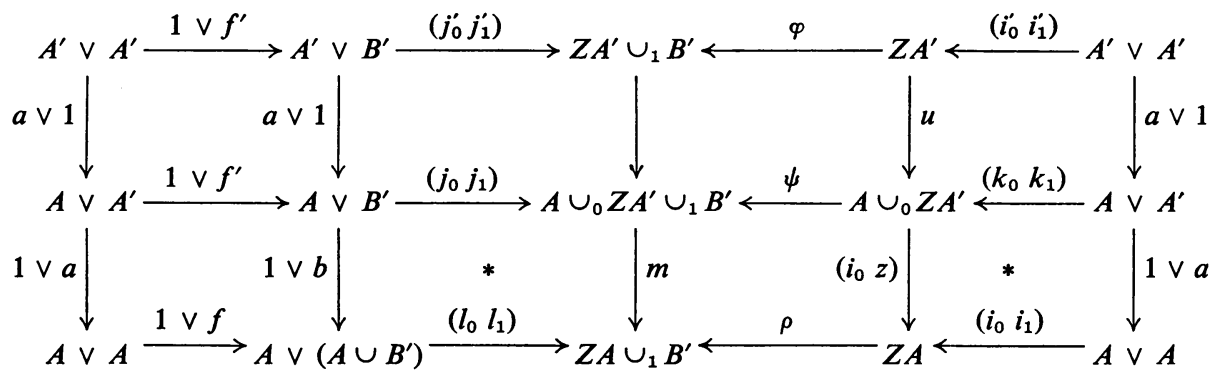

Notice that the first and last columns are identical and that the three rows are 
consequently squares. The top row is the pushout of $\left(i_{0}^{\prime} i_{1}^{\prime}\right)$ and $1 \vee f^{\prime}$, the former being a cofibration; the four squares immediately under this are pushouts as well, $u$, as a pushout of $a \vee 1$, being a cofibration, and the second row is accordingly a pushout. The unstarred squares in the next row are again pushouts (the right-hand starred square having already been defined), and it follows that the bottom row is a pushout.

Now in the square represented by the first row we observe that $j_{1}^{\prime}$ is the pushout of $i_{1}^{\prime}$ along $f^{\prime}$ and is thus, by $\mathrm{HC} 3$, a homotopy equivalence. Since $\varphi i_{1}^{\prime}=j_{1}^{\prime} f^{\prime}$ and $j_{0}^{\prime}=\varphi i_{0}^{\prime}$ we conclude that $\varphi$ and $j_{0}^{\prime}$ are homotopy equivalences.

By analogous arguments $k_{0}, j_{0}$ and $\psi$ are homotopy equivalences. Since $\left(i_{0} z\right) k_{0}$ $=i_{0},\left(i_{0} z\right)$ is a homotopy equivalence as well as a cofibration and HC3 permits the same conclusion about $m$. But $m \psi=\rho\left(i_{0} z\right)$ so that $\rho$ too is a homotopy equivalence.

In the bottom row we observe that $l_{1}$ is the pushout of $i_{1}$ along $f$ so that it too is a homotopy equivalence. Finally, $l_{1} f=\rho i_{1}$, and the conclusion follows.

LEMMA 1.3. If in the commutative diagram

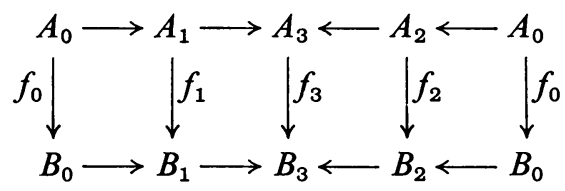

the rows are pushouts with morphisms in $\operatorname{Cof} \mathscr{C}$, and if $f_{0}, f_{1}, f_{2}$ are homotopy equivalences, then $f_{3}$ is a homotopy equivalence.

To see this, we construct the diagram

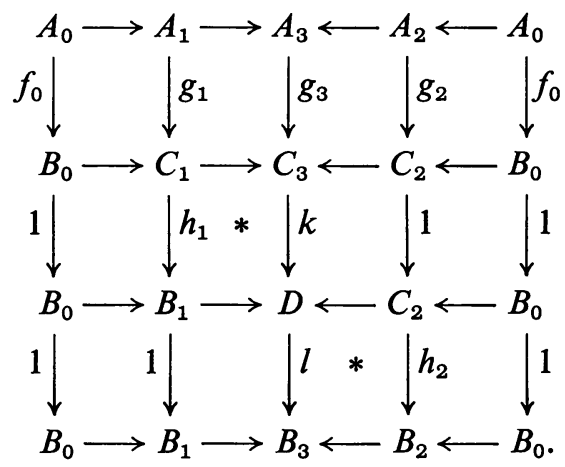

The squares under the top row are pushouts; this makes the second row a pushout and defines $h_{\alpha}: C_{\alpha} \rightarrow B_{\alpha}$ such that $h_{\alpha} g_{\alpha}=f_{\alpha}, \alpha=1,2$. The square marked $*$ under the second row is a pushout, so that the third row is a pushout. Finally, the square marked $*$ under the third row is a pushout. Commutativity of the diagram implies $l k g_{3}=f_{3}$.

By $1.2, g_{1}$ and $g_{2}$ are homotopy equivalences, hence also $g_{3}, k$ and $l$. The conclusion follows. 
We shall also need below some notions concerning relative homotopy. If $a: A^{\prime} \rightarrow A$ is a cofibration, we construct the pushout

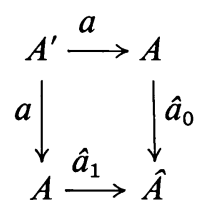

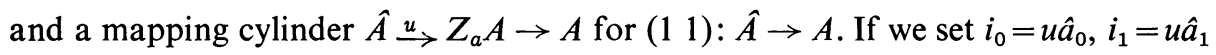
then $\left(i_{0} i_{1}\right): A \vee A \rightarrow Z_{a} A$ is a relative cylinder over $A$ (rel $\left.a\right)$. We say that $f_{0}, f_{1}$ : $A \rightarrow X$ are homotopic (rel $a$ ) if there is an $F: Z_{a} A \rightarrow X$ with $F i_{0}=f_{0}, F i_{1}=f_{1}$.

It is easy to see that this relation has all the usual properties; e.g., that it is an equivalence relation in $\mathscr{C}(A, X)$.

LEMma 1.4. Consider the c-pushout 1.1. If $g_{0}, g_{1}: A \rightarrow X$ are homotopic (rel $a$ ) and $v: B^{\prime} \rightarrow X$ satisfies $v u=g_{0}$ a then $\left(g_{0} v\right) \simeq\left(g_{1} v\right): A \cup B^{\prime} \rightarrow X$.

LemMA 1.5. Let $a: A^{\prime} \rightarrow A$ be a cofibration. Suppose that the square in the commutative diagram

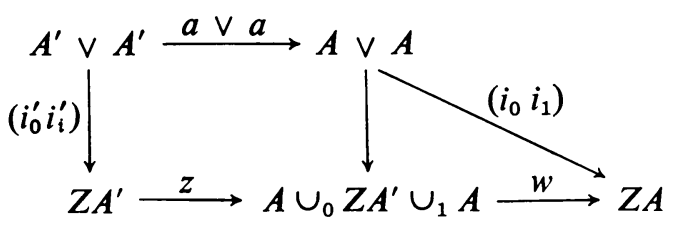

is a pushout, that $w$ is a cofibration, and that $\left(i_{0}^{\prime} i_{1}^{\prime}\right),\left(i_{0} i_{1}\right)$ are cylinders. If $F: Z A \rightarrow X$, $f_{1}: A \rightarrow X$ and $f_{1} \simeq F i_{1}(\operatorname{rel} a)$ then there is $a G: Z A \rightarrow X$ such that $G w z=F w z$, $G i_{0}=F i_{0}$ and $G i_{1}=f_{1}$.

The proof proceeds by taking for $X$ the object $Z A+Z_{a} A$ in the pushout

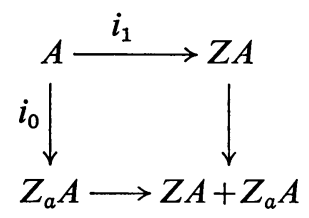

and using homotopy extension.

2. $\mathscr{L}$-lattices. By an $\mathscr{L}$-lattice we mean a distributive lattice in which every element has only finitely many predecessors. Such a lattice has of course a smallest element 0 . We define the category $\mathscr{L}$, whose objects are $\mathscr{L}$-lattices, by taking as morphisms the maps $f: \Lambda \rightarrow \Gamma$ which preserve the lattice operations and are initial; i.e., have the property that if $\gamma \leqq f \lambda$ then $\gamma \in f(\Lambda)$. We may occasionally want to consider maps $\Lambda \rightarrow \Gamma$ which are not morphisms in $\mathscr{L}$; properly signalled, they should lead to no confusion. 
We shall say that $f: \Lambda \rightarrow \Gamma$ is a cofibration if $f \lambda \leqq f \lambda^{\prime}$ implies $\lambda \leqq \lambda^{\prime}$; notice that this makes $f$ injective. We shall see presently that $\mathscr{L}$, equipped with the class Cof $\mathscr{L}$ of cofibrations, is a c-category.

LEMMA 2.1. If $f: \Lambda \rightarrow \Gamma$ is a cofibration in $\mathscr{L}$ then $f^{*} \gamma=\bigvee\{\lambda \mid f \lambda \leqq \gamma\}$ defines a morphism in $\mathscr{L}$, such that $f^{*} f=1_{\Lambda}$.

We remark parenthetically that if we regard ordered sets as categories in the usual way then $f$ becomes a functor and $f^{*}$ is adjoint to $f$.

LemMA 2.2. Every morphism in $\mathscr{L}$ factors as $g f$ where $f$ is a surjective morphism and $g$ is a cofibration.

Proposition 2.3. ( $\mathscr{L}$, Cof $\mathscr{L})$ is a c-category.

Axiom $\mathrm{C} 1$ is clear. For $\mathrm{C} 2$ we must show the existence of a pushout

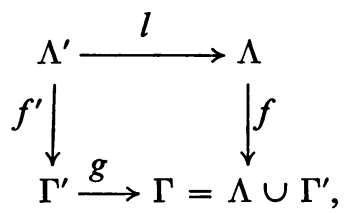

with $g$ a cofibration, whenever $l$ is a cofibration. In view of 2.2 , it is sufficient to consider the two cases, that $f^{\prime}$ is surjective and that $f^{\prime}$ is a cofibration.

In the former case we have two congruences on $\Lambda$ :

$$
\begin{array}{ll}
\lambda \sim_{1} \mu & \text { if and only if } f^{\prime} l^{*} \lambda=f^{\prime} l^{*} \mu ; \\
\lambda \sim_{2} \mu & \text { if and only if }(\lambda \wedge \mu) \vee l^{*} \lambda=\lambda \text { and }(\lambda \wedge \mu) \vee l^{*} \mu=\mu .
\end{array}
$$

To see that the second relation is transitive we suppose also $\mu_{2} \nu$ and observe that

$$
\lambda=(\lambda \wedge \mu) \vee l^{*} \lambda=\lambda \wedge\left((\mu \wedge \nu) \vee l^{*} \mu\right) \vee l^{*} \lambda=(\lambda \wedge \mu \wedge \nu) \vee l^{*} \lambda .
$$

The intersection $\sim$ of $\sim_{1}$ and $\sim_{2}$ is again a congruence and has the property that if $\lambda^{\prime}, \mu^{\prime} \in \Lambda^{\prime}$ then $l \lambda^{\prime} \sim l \mu^{\prime}$ if and only if $f^{\prime} \lambda^{\prime}=f^{\prime} \mu^{\prime}$. We need only take $\Gamma$ to be the quotient of $\Lambda$ by $\sim$.

In the latter case, we set $\Gamma=\left\{\left(\lambda, \gamma^{\prime}\right) \mid l^{*} \lambda=f^{*} \gamma^{\prime}\right\} \subset \Lambda \times \Gamma^{\prime}$. Now $\Lambda \times \Gamma^{\prime}$, with the usual ordering, is again an $\mathscr{L}$-lattice; it is in fact both the product and the coproduct of $\Lambda$ and $\Gamma^{\prime}$. It is easy to see that $\Gamma$ is a sublattice of $\Lambda \times \Gamma^{\prime}$ and that $f: \Lambda \rightarrow \Gamma, g: \Gamma^{\prime} \rightarrow \Gamma$, defined by $f \lambda=\left(\lambda, f^{\prime} l^{*} \lambda\right), g \gamma^{\prime}=\left(l f^{\prime *} \gamma^{\prime}, \gamma^{\prime}\right)$ are cofibrations in $\mathscr{L}$. An easy computation shows that the square is a pushout.

3. c-systems. We regard ordered sets as categories. If $\Lambda$ is an ordered set and $F: \Lambda \rightarrow \mathscr{C}$ is a functor we write $F_{\lambda}$ for the value of $F$ at $\lambda \in \Lambda$ and $F_{\mu}^{\lambda}: F_{\lambda} \rightarrow F_{\mu}$ for the value of $F$ on the unique morphism $\lambda \rightarrow \mu$ when $\lambda \leqq \mu$. 
If $(\mathscr{C}, \operatorname{Cof} \mathscr{C})$ is a c-category and $\Lambda$ is a $\mathscr{L}$-lattice, a c-system in $\mathscr{C}$ with indexlattice $\Lambda$ is a functor $F: \Lambda \rightarrow \mathscr{C}$ such that all $F_{\mu}^{\lambda}$ are cofibrations, $F_{0}=0$, and, for any $\lambda, \mu \in \Lambda$

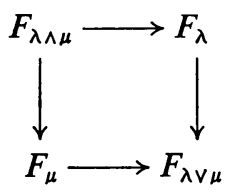

is a pushout. The full subcategory of the category of functors $\Lambda \rightarrow \mathscr{C}$ containing the c-cystems is written $c(\Lambda, \mathscr{C})$, so that $c(\Lambda, \mathscr{C})$ becomes a c-category. For this purpose we introduce the notion of a 2-cofibration in $\mathscr{C}$ (cf. $[6, \S 18])$, viz. a commutative square

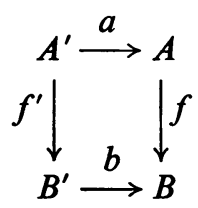

such that $a$ and ( $f b): A \cup B^{\prime} \rightarrow B$ (equivalently, $f^{\prime}$ and $(f b)$ ) are cofibrations, the existence of $A \cup B^{\prime}$ being guaranteed by $\mathrm{C} 2$.

LEMMA 3.1. If each of the two squares

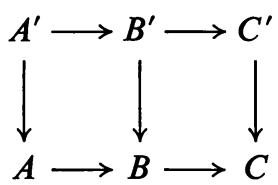

is a 2-cofibration, then so is the rectangle.

LEMMA 3.2. If in the diagram

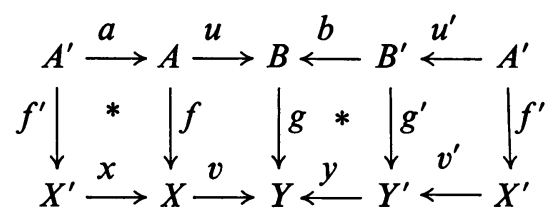

the top row $\left({ }^{3}\right)$ is a 2-cofibration and the starred squares are pushouts then the bottom row is a 2-cofibration.

${ }^{(3)}$ Compare 1.2 for notation. 
For $x$ and $y$ are cofibrations by C2. It is not difficult to see that

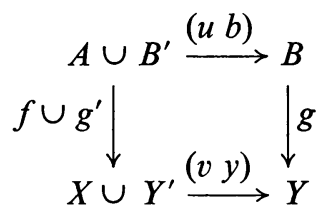

is a pushout, so that $\left(\begin{array}{ll}v & y\end{array}\right)$ is again a cofibration.

We now define $\operatorname{Cof} c(\Lambda, \mathscr{C})$ as the class of morphisms $f: F \rightarrow G$ such that for all $\lambda \leqq \mu$

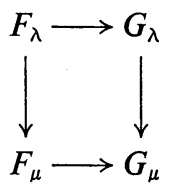

is a 2-cofibration.

Proposition 3.3. $(c(\Lambda, \mathscr{C})$, $\operatorname{Cof} c(\Lambda, \mathscr{C}))$ is a c-category.

There is really only one point which needs checking. Suppose $f: F^{\prime} \rightarrow F$ is a cofibration and $u^{\prime}: F^{\prime} \rightarrow G^{\prime}$ is any morphism in $c(\Lambda, \mathscr{C})$. Then $G: \Lambda \rightarrow \mathscr{C}$ may be defined by taking $G_{\lambda}$ to be the pushout in

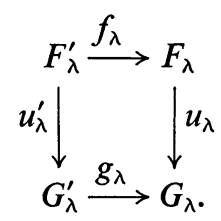

It is easily verified that $G$ is a c-system and that the corresponding diagram in $c(\Lambda, \mathscr{C})$ is a pushout. That $g$ is a cofibration follows from 3.2 .

If $F: \mathscr{C} \rightarrow \mathscr{C}^{\prime}$ is a c-functor then $c(\Lambda, F): c(\Lambda, \mathscr{C}) \rightarrow c\left(\Lambda, \mathscr{C}^{\prime}\right)$, composition with $F$, is also a c-functor. If $l: \Lambda^{\prime} \rightarrow \Lambda$ is a lattice map then $c(l, \mathscr{C}): c(\Lambda, \mathscr{C}) \rightarrow c\left(\Lambda^{\prime}, \mathscr{C}\right)$, composition with $l$, is a c-functor.

We are now in a position to define c-completeness. A c-category $\mathscr{C}$ is c-complete if any c-system in $\mathscr{C}$ has a colimit, and if all the functors colim: $c(\Lambda, \mathscr{C}) \rightarrow \mathscr{C}$ whose existence is thus assured preserve cofibrations. As coadjoints they must preserve pushouts and are accordingly c-functors.

Proposition 3.4. $\mathscr{L}$ is c-complete.

The colimit of a c-system $\Phi: \Lambda \rightarrow \mathscr{L}$ is the colimit of the underlying sets, provided with the weakest ordering such that all injections are order preserving.

\section{Prolongations of c-systems.}

LeMmA 4.1. If $l: \Lambda \rightarrow \Gamma$ is a cofibration in $\mathscr{L}$ and $\mathscr{C}$ is a c-category then $c(l, \mathscr{C})$ : $c(\Gamma, \mathscr{C}) \rightarrow c(\Lambda, \mathscr{C})$ is adjoint to $c\left(l^{*}, \mathscr{C}\right): c(\Lambda, \mathscr{C}) \rightarrow c(\Gamma, \mathscr{C})$, and for any $A \in c(\Gamma, \mathscr{C})$ the junction $u:$ All ${ }^{*} \rightarrow A$ is a cofibration in $c(\Gamma, \mathscr{C})$. 
The morphism $u$ is given by $u_{\gamma}=A_{\gamma}^{l * \gamma}$; the conclusion is immediate.

LEMMA 4.2. If

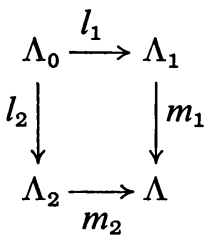

is a pushout in $\mathscr{L}$, with all morphisms cofibrations, and $F_{i}: \Lambda_{i} \rightarrow \mathscr{C}$ are c-systems such that $F_{1} l_{1}=F_{2} l_{2}=F_{0}$ then there is a c-system $F: \Lambda \rightarrow \mathscr{C}$ such that $F m_{i}=F_{i}$. If also $G: \Lambda \rightarrow \mathscr{C}$ is a c-system and $f_{i}: F_{i} \rightarrow G m_{i}, i=1,2$ satisfy $f_{1} l_{1}=f_{2} l_{2}$ then there is a unique $f: F \rightarrow G$ such that $f m_{i}=f_{i}$.

We need only define $F$ by the pushout

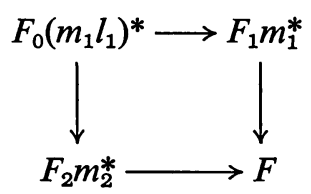

in $c(\Gamma, \mathscr{C})$.

If we write $[n]$ for the set $\{0,1, \ldots, n\}$ provided with its usual ordering then $[n] \in \mathscr{L}$; we see easily that for any c-category $\mathscr{C}, c([0], \mathscr{C})=0, c([1], \mathscr{C}) \approx \mathscr{C}$. The category $c([2], \mathscr{C})$ has as its objects the cofibrations of $\mathscr{C}\left({ }^{4}\right)$.

Applying 4.2 to $c([2], \mathscr{C})$ we get the following corollary.

COROLlaRY 4.3. In the situation of 4.2 , if $a_{1}, a_{2}$ are cofibrations in $c\left(\Lambda_{1}, \mathscr{C}\right)$, $c\left(\Lambda_{2}, \mathscr{C}\right)$ such that $a_{1} l_{1}=a_{2} l_{2}$ then there is a unique $a \in c(\Lambda, \mathscr{C})$ with am $m_{i}=a_{i}$, and $a$ is again a cofibration.

5. Premorphisms. We shall want to study morphisms between colimits of c-systems. We introduce for this purpose the following calculus of "premorphisms".

Suppose $\Lambda, \Gamma \in \mathscr{L}$, and that $A: \Lambda \rightarrow \mathscr{A}, B: \Gamma \rightarrow \mathscr{A}$ are any functors. By a premorphism from $A$ to $B$ we mean a pair $(\varphi, f)$ where $\varphi: \Lambda \rightarrow \Gamma$ is $\vee$-preservinghence preserving order and 0 , but not necessarily in $\mathscr{L}$-and $f$ is a morphism $f: A \rightarrow B \varphi$ in the category of functors $\Lambda \rightarrow \mathscr{A}$.

If also $C: \Delta \rightarrow \mathscr{A}$ and $(\theta, g): B \rightarrow C$ is a premorphism then so is

$$
(\theta \varphi, g \circ f): A \rightarrow C, \quad \text { where } g \circ f=(g \varphi) f .
$$

If $(\varphi, f): A \rightarrow B$ is a premorphism, $\psi: \Lambda \rightarrow \Gamma$ is $\vee$-preserving and $\psi \geqq \varphi$, i.e., $\psi \lambda \geqq \varphi \lambda$ for all $\lambda \in \Lambda$, then $\left(\psi, f^{\psi}\right)$ is again a premorphism from $A$ to $B$, where $f_{\lambda}^{\psi}=B_{\psi \lambda}^{\varphi \lambda} f_{\lambda}$.

$\left.{ }^{4}\right)$ In the notation of [6], $c([2], \mathscr{C})$ was $\operatorname{Cof} \mathscr{C}$. 
Now suppose $A$ and $B$ have colimits in $\mathscr{A}$. Then a premorphism $(\varphi, f): A \rightarrow B$ defines a morphism $[\varphi, f]: \operatorname{colim} A \rightarrow \operatorname{colim} B$ by the rule $[\varphi, f] \operatorname{inj}_{\lambda}=\operatorname{inj}_{\varphi \lambda} f_{\lambda}$. If also $C$ has a colimit and $(\theta, g): B \rightarrow C$ is a premorphism then $[\theta, g][\varphi, f]=[\theta \varphi, g \circ f]$. Finally, if $\psi \geqq \varphi$ then $\left[\psi, f^{\psi}\right]=[\varphi, f]$.

A special case is that in which $\Lambda=\Gamma$. A pair $(1, f)$ is a premorphism precisely when $f: A \rightarrow B$ is a morphism is the functor category, and $[1, f]=\operatorname{colim} f$.

If $B: \Gamma \rightarrow \mathscr{A}$ has a colimit and $X \in \mathscr{A}$ we say that $X$ is compact relative to $B$ if colim $\mathscr{A}(X, B) \rightarrow \mathscr{A}(X$, colim $B)$ is an isomorphism.

LEMMA 5.1. If $A: \Lambda \rightarrow \mathscr{A}$ and $B: \Gamma \rightarrow \mathscr{A}$ have colimits and each $A_{\lambda}$ is compact relative to $B$ then any morphism colim $A \rightarrow \operatorname{colim} B$ is of the form $[\varphi, f]$ for some premorphism $(\varphi, f): A \rightarrow B$. Furthermore, $[\varphi, f]=[\psi, g]$ if and only if for some $\theta \geqq \varphi, \psi, f^{\theta}=g^{\theta}$.

If $w:$ colim $A \rightarrow \operatorname{colim} B$ then for each $\lambda \in \Lambda$ there is a $\varphi_{0} \lambda \in \Gamma$ and an $f_{\lambda}^{0}: A_{\lambda} \rightarrow$ $B_{\varphi_{0} \lambda}$ such that $\varphi_{0} 0=0$ and $w \operatorname{inj}_{\lambda}=\operatorname{inj}_{\varphi_{0} \lambda} f_{\lambda}^{0}$. Since $\lambda$ has only finitely many predecessors there is a $\varphi_{1} \lambda \geqq \bigvee_{\mu \leqq \lambda} \varphi_{0} \mu$ such that $\varphi_{1} 0=0$ and, for all $\mu \leqq \lambda$,

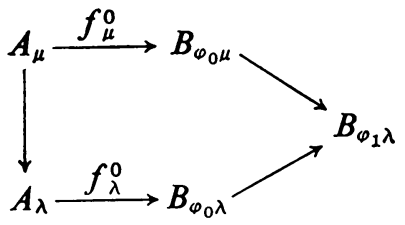

commutes. If we set $\varphi \lambda=\bigvee_{\mu \leqq \lambda} \varphi_{1} \mu$ then $\varphi: \Lambda \rightarrow \Gamma$ is $\vee$-preserving. If $f_{\lambda}=B_{\varphi \lambda}^{\varphi_{0} \lambda} f_{\lambda}^{0}$ then $(\varphi, f)$ is a premorphism and $[\varphi, f]=w$.

If $[\varphi, f]=[\psi, g]$ then for each $\lambda \in \Lambda$ there is a $\theta_{0} \lambda \in \Gamma$ such that $\theta_{0} 0=0, \theta_{0} \lambda \geqq$ $\varphi \lambda \vee \psi \lambda$, and $B_{\theta_{0} \lambda}^{\infty \lambda} f_{\lambda}=B_{\theta_{0} \lambda}^{\psi \lambda} g_{\lambda}$. If $\theta \lambda=\bigvee_{\mu \leqq \lambda} \theta_{0} \mu$ then $\theta$ is $\vee$-preserving and $f^{\theta}=g^{\theta}$.

LEMMA 5.2. Under the hypothesis of 5.1 there is, for any morphism

$$
w: \operatorname{colim} A \rightarrow \operatorname{colim} B,
$$

a cofibration $j: \Gamma \rightarrow \Delta$ in $\mathscr{L}$, a cofinal lattice map $p: \Delta \rightarrow \Lambda$ and a morphism $f: A p \rightarrow B j^{*}$ such that $w=\operatorname{colim} f$.

We call such an $f$ a realization of $w$.

Let $(\varphi, g): A \rightarrow B$ be a premorphism representing $w$ and let $\Delta \subset \Lambda \times \Gamma$ be the sublattice $\{(\lambda, \gamma) \mid \gamma \geqq \varphi \lambda\} . p: \Delta \rightarrow \Lambda$ is the restriction of the projection. If $j \gamma=(0, \gamma)$ then $j$ is a cofibration, and $j^{*}$ is in fact the restriction of the projection $\Delta \rightarrow \Gamma$. We need only set $f_{(\lambda, \gamma)}=B_{\lambda}^{\varphi \gamma} g_{\lambda}$.

Lemma 5.2 will be useful in the case that $\mathscr{A}$ is a c-category and $A$ and $B$ are c-systems. For then, since $p$ and $j^{*}$ are lattice maps, $A p$ and $B_{j *}$ are again c-systems and $f$ is a morphism in $c(\Delta, \mathscr{A})$.

6. c-completions I: Definition. We shall say that $\mathscr{C}$ is a c-completion of $\mathscr{C}_{0}$ if $\mathscr{C}_{0}$ is a small c-subcategory of $\mathscr{C}$ such that 
(i) every c-system in $\mathscr{C}_{0}$ has a colimit in $\mathscr{C}$ and the functors colim: $c\left(\Lambda, \mathscr{C}_{0}\right) \rightarrow \mathscr{C}$ which are consequently defined are c-functors,

(ii) every $A \in \mathscr{C}_{0}$ is compact with respect to c-systems in $\mathscr{C}_{0}$,

(iii) every object (cofibration) in $\mathscr{C}$ is a colimit of an object (cofibration) in some $c\left(\Lambda, \mathscr{C}_{0}\right)$.

It follows immediately from 5.2 that every morphism in $\mathscr{C}$ is a colimit of a morphism in some $c\left(\Lambda, \mathscr{C}_{0}\right)$.

Condition (iii) gives a representation for cofibrations in $\mathscr{C}$. We may obtain an alternative one as follows. By 4.1 if $A^{\prime}$ is the restriction of a c-system $A$ to an initial sublattice then colim $A^{\prime} \rightarrow \operatorname{colim} A$ is a cofibration. The converse is also true.

Lemma 6.1. If $\mathscr{C}$ is a c-completion of $\mathscr{C}_{0}, A \in c\left(\Gamma, \mathscr{C}_{0}\right)$ and $w:$ colim $A \rightarrow X$ is a cofibration then there is a $\Delta \in \mathscr{L}$ containing $\Gamma$ as an initial sublattice, a prolongation $\bar{A}$ of $A$ to a c-system on $\Delta$ and an isomorphism colim $\bar{A} \rightarrow X$ such that $w$ is the composition colim $A \rightarrow \operatorname{colim} \bar{A} \rightarrow X$.

By condition (iii) we may suppose there is a cofibration $b: B^{\prime} \rightarrow B$ in some $c\left(\Lambda, \mathscr{C}_{0}\right)$ with colim $B^{\prime}=A$ and colim $b=w$. By 5.2 there is a realization $g: B^{\prime} p \rightarrow A j^{*}$ in $c\left(\Delta, \mathscr{C}_{0}\right)$ of the identity morphism of colim $A$, where $j: \Gamma \rightarrow \Delta$ is the inclusion of an initial sublattice. Let

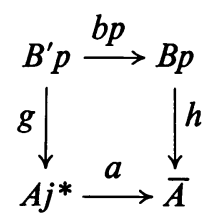

be a pushout in $c\left(\Delta, \mathscr{C}_{0}\right)$. Then, since colim preserves pushouts, colim $h$ is an isomorphism. But colim $A=\operatorname{colim} A j^{*}=\operatorname{colim} B^{\prime} p=\operatorname{colim} B$, and colim $B p=\operatorname{colim} B$ $=X$.

LEMMA 6.2. If $\mathscr{C}$ is a c-completion of $\mathscr{C}_{0}$ then any 2-cofibration in $\mathscr{C}$ is the colimit of a 2-cofibration in some $c\left(\Delta, \mathscr{C}_{0}\right)$.

By 6.1 we may suppose that the 2-cofibration in $\mathscr{C}$ is of the form

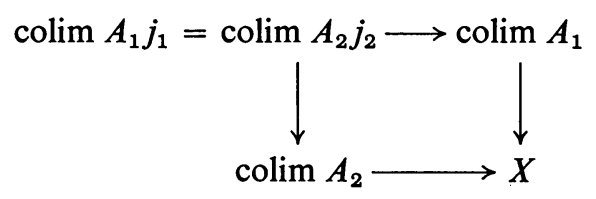

where $j_{1}: \Lambda \rightarrow \Lambda_{1}$ and $j_{2}: \Lambda \rightarrow \Lambda_{2}$ are cofibrations in $\mathscr{L}, A_{\alpha} \in c\left(\Lambda_{\alpha}, \mathscr{C}_{0}\right)$ and 
$A_{1} i_{1}=A_{2} i_{2}$. If

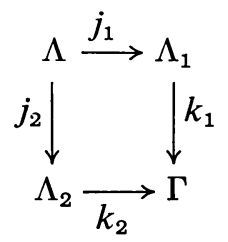

is a pushout in $\mathscr{L}$ then, by $4.2, A_{1}$ and $A_{2}$ have a common extension $A \in c\left(\Gamma, \mathscr{C}_{0}\right)$. But then colim $A$ is the pushout in 6.3 and the morphism colim $A \rightarrow X$ is a cofibration. Applying 6.1 once more, $X$ may be replaced by $\operatorname{colim} B$ where $B$ is a prolongation of $A$ to some $\Delta$ containing $\Gamma$ as an initial sublattice. If $d: \Gamma \rightarrow \Delta$ is the inclusion then

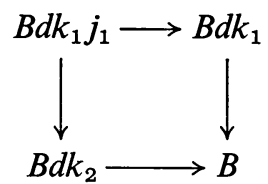

is a 2 -cofibration in $c\left(\Delta, \mathscr{C}_{0}\right)$ having 6.3 as a colimit.

COROllaRy 6.4. If $\mathscr{C}$ is a c-completion of $\mathscr{C}_{0}$ then $c([2], \mathscr{C})$ is a c-completion of $c\left([2], \mathscr{C}_{0}\right)$.

7. c-completions II: Completeness and uniqueness. If $\mathscr{C}$ is a c-category and $\Phi: \Lambda \rightarrow \mathscr{L}$ is a c-system then a family $f_{\lambda}, \lambda \in \Lambda$ of c-systems $A^{\lambda}: \Phi_{\lambda} \rightarrow \mathscr{C}$ is coherent if for each $\lambda \leqq \mu, A^{\mu} \Phi_{\mu}^{\lambda}=A^{\lambda}$. If each of the colimits $A_{\lambda}^{\Phi}=$ colim $A^{\lambda}$ exists then, by 4.1, 4.2, $A^{\Phi}: \Lambda \rightarrow \mathscr{C}$ is a c-system.

If $\mathscr{C}$ is a c-completion of $\mathscr{C}_{0}$ and, for all $\lambda, A^{\lambda}$ has its values in $\mathscr{C}_{0}$, then $A^{\Phi}$ exists. Furthermore, $\hat{A}$ : colim $\Phi \rightarrow \mathscr{C}_{0}$, defined by $\hat{A} \operatorname{inj}_{\lambda}=A^{\lambda}$, is again a c-system.

In this case $A^{\Phi}$ has an obvious colimit in $\mathscr{C}$, viz. colim $\hat{A}$. We shall use this fact to deduce the c-completeness of $\mathscr{C}$.

LEMMA 7.1. If $\mathscr{C}$ is a c-completion of $\mathscr{C}_{0}$ and $B: \Lambda \rightarrow \mathscr{C}$ is a c-system then there is $a$ c-system $\Phi: \Lambda \rightarrow \mathscr{L}$ and a coherent family of c-systems $A^{\lambda}: \Phi_{\lambda} \rightarrow \mathscr{C}_{0}$ such that $A^{\Phi} \approx B$

We consider partial systems $(\Gamma, \Phi, A, \varphi)$, where $\Gamma$ is an initial sublattice of $\Lambda$, $\Phi: \Gamma \rightarrow \mathscr{L}$ is a c-system, $A$ is a coherent family of c-systems $A^{\lambda}: \Phi_{\lambda} \rightarrow \mathscr{C}_{0}$ and $\varphi: A^{\Phi} \approx B \mid \Gamma$. These are ordered in the obvious way by extension; Zorn's lemma then applies. Thus we may take $(\Gamma, \Phi, A, \varphi)$ maximal with respect to extensions. We must show that $\Gamma=\Lambda$.

Suppose this is not the case. Then there is a minimal $\lambda \in \Lambda-\Gamma$. We shall show that $(\Gamma, \Phi, A, \varphi)$ can be extended to the sublattice $\Gamma^{\prime}$ generated by $\Gamma$ and $\lambda$. Let $\gamma \in \Gamma$ be the largest predecessor of $\lambda$, i.e., the image of $\lambda$ under the adjoint of the 
inclusion $\Gamma \subset \Gamma^{\prime}$. By 6.1 there is a cofibration $\Phi_{\lambda}^{\gamma}: \Phi_{\gamma} \rightarrow \Phi_{\lambda}$ in $\mathscr{L}$, a c-system $A^{\lambda}: \Phi_{\lambda} \rightarrow \mathscr{C}_{0}$ such that $A^{\lambda} \Phi_{\lambda}^{\gamma}=A^{\gamma}$ and an isomorphism $\varphi_{\lambda}$ such that

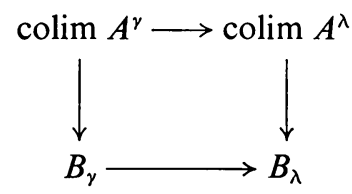

commutes.

Now $\Gamma^{\prime}$ is the pushout in $\mathscr{L}$ of $\Gamma$ and $\left\{\lambda^{\prime} \mid \lambda^{\prime} \leqq \lambda\right\}$. Thus, by $4.2, \Phi$ extends uniquely to a c-system on $\Gamma^{\prime}$ and, again by 4.2 , since each $\Phi_{\gamma^{\prime}}$ is itself a pushout, $A$ extends uniquely to a coherent system on $\Gamma^{\prime}$. But each new $A_{\gamma^{\prime}}^{\Phi}$ is again a pushout; thus morphisms $\varphi_{\gamma^{\prime}}: A_{\gamma^{\prime}}^{\Phi} \rightarrow B_{\gamma^{\prime}}$ are uniquely defined by naturality and are isomorphisms.

Proposition 7.2. If $\mathscr{C}$ is a c-completion of $\mathscr{C}_{0}$ then $\mathscr{C}$ is c-complete. Further, every object of $\mathscr{C}_{0}$ is compact with respect to all c-systems in $\mathscr{C}$.

It follows immediately from 7.1 that every c-system in $\mathscr{C}$ has a colimit. To see that colim: $c(\Lambda, \mathscr{C}) \rightarrow \mathscr{C}$ preserves cofibrations and is thus a c-functor, we apply 7.1 to the category $c([2], \mathscr{C})$ which by 6.4 is a c-completion of $c\left([2], \mathscr{C}_{0}\right)$ : a cofibration in $c(\Lambda, \mathscr{C})$ is an object of $c([2], c(\Lambda, \mathscr{C}))=c(\Lambda, c([2], \mathscr{C}))$.

The compactness of the objects of $\mathscr{C}_{0}$ is, once more, a consequence of 7.1 together with condition (ii) for c-completions.

A functor from one c-category to another is c-continuous if it is a c-functor which preserves colimits of c-systems. A c-completion enjoys the following universal property with respect to c-continuous functors.

Proposition 7.3. If $\mathscr{C}$ is a c-completion of $\mathscr{C}_{0}, \mathscr{D}$ is a c-complete c-category and $F_{0}: \mathscr{C}_{0} \rightarrow \mathscr{D}$ is a c-functor then $F_{0}$ has an extension to a c-continuous functor $F: \mathscr{C} \rightarrow \mathscr{D}$. If $F^{\prime}$ similarly extends $F_{0}^{\prime}: \mathscr{C}_{0} \rightarrow \mathscr{D}$ and $f_{0}: F_{0} \rightarrow F_{0}^{\prime}$ then $f_{0}$ has a unique extension to a morphism $f: F \rightarrow F^{\prime}$.

This implies that the extension $F$ of $F_{0}$ is essentially unique.

To construct $F$ we associate to each object $X \in \mathscr{C}$ a c-system $A_{X} \in c\left(\Lambda_{X}, \mathscr{C}_{0}\right)$ of which it is the colimit. We may do this in such a way that if $X \in \mathscr{C}_{0}$ then $\Lambda_{X}=\{0\}$. Then we must set $F X=\operatorname{colim} F_{0} A_{X}$. If $(\varphi, a): A_{X} \rightarrow A_{Y}$ is a premorphism then so is $\left(\varphi, F_{0} a\right): F_{0} A_{X} \rightarrow F_{0} A_{Y}$. If $[\varphi, a]=[\psi, b]$ then, by $5.1,\left[\varphi, F_{0} a\right]=\left[\psi, F_{0} b\right]$. The results of $\S 5$ show further that $[\varphi, a] \rightarrow\left[\varphi, F_{0} a\right]$ preserves composition, so that $F[\varphi, a]=\left[\varphi, F_{0} a\right]$ defines a functor $F: \mathscr{C} \rightarrow \mathscr{D}$.

Suppose $\left\{A_{X}^{\prime}\right\}$ is another family of colimit representations. Then for each $X \in \mathscr{C}$ there is a premorphism $\left(\theta_{X}, u_{X}\right): A_{X} \rightarrow A_{X}^{\prime}$ with $\left[\theta_{X}, u_{X}\right]=1_{X}$. If we set $f_{X}=\left[1, f_{0}\right]$ . $\left[\theta_{X}, u_{X}\right]$ then $f$ is the required extension of $f_{0}$. In particular, up to canonical isomorphism, $F$ is independent of the choice of $\left\{A_{X}\right\}$. It is clear from its definition that $F$ is a c-functor; Lemma 7.1 shows that it preserves colimits of c-systems. 
This proposition has as a corollary the uniqueness theorem for c-completions:

THEOREM 7.4. Any two c-completions of a c-category are equivalent via an extension of the identity; the equivalence itself is unique up to canonical isomorphism.

In view of this theorem we may permit ourselves to speak of the c-completion of a c-category.

The following example should be kept in mind. Let $\mathscr{C}_{0}$ be the category of pointed finite $\mathrm{CW}$-complexes and cellular maps. Cofibrations are maps isomorphic to the inclusion of a subcomplex. Then the c-completion of $\mathscr{C}_{0}$ is the category of all pointed $\mathrm{CW}$-complexes.

8. c-completions III: Existence. We concern ourselves next with the existence of c-completions. We shall see first that if a small c-category $\mathscr{C}_{0}$ has a c-completion $\mathscr{C}$ then $\mathscr{C}$ is equivalent to a full subcategory of the category $\mathscr{F}$ of functors $\mathscr{C}_{0}^{\text {op }} \rightarrow \mathscr{S}$, where $\mathscr{S}$ is the category of pointed sets.

Let $R: \mathscr{C} \rightarrow \mathscr{F}$ be the representing functor, $(R X) A=\mathscr{C}(A, X)$. Then $R$ takes colimits of c-systems in $\mathscr{C}$ into colimits in $\mathscr{F}$. In particular, if $B \in c\left(\Lambda, \mathscr{C}_{0}\right)$ then $R($ colim $B)=$ colim $\mathscr{C}_{0}(-, B)$. Functors of the latter form will be called c-accessible -notice that they are defined for any small c-category.

LEMma 8.1. If $\mathscr{C}$ is a c-completion of $\mathscr{C}_{0}$ then $R$ is an equivalence of $\mathscr{C}$ with the full subcategory of $\mathscr{F}$ containing the c-accessible functors.

This is just Yoneda's lemma. If $B \in c\left(\Lambda, \mathscr{C}_{0}\right)$ and $C \in c\left(\Gamma, \mathscr{C}_{0}\right)$ then

$$
\begin{aligned}
\mathscr{F}\left(\operatorname{colim}_{\Lambda} \mathscr{C}_{0}(-, B), \operatorname{colim}_{\Gamma} \mathscr{C}_{0}(-, C)\right) & \approx \lim _{\Lambda} \operatorname{colim}_{\Gamma} \mathscr{F}\left(\mathscr{C}_{0}(-, B), \mathscr{C}_{0}(-, C)\right) \\
& \approx \lim _{\Lambda} \operatorname{colim}_{\Gamma} \mathscr{C}_{0}(B, C) \\
& \approx \mathscr{C}\left(\operatorname{colim}_{\Lambda} B, \operatorname{colim}_{\Gamma} C\right) .
\end{aligned}
$$

It is now clear what is the candidate for the c-completion of a small c-category: $\mathscr{C}_{0}$ being such a category let us write $\mathscr{C}$ for the full subcategory of $\mathscr{F}$ containing the c-accessible functors and identify $\mathscr{C}_{0}$ with the subcategory of representable functors in $\mathscr{C}$.

We propose to show that $\mathscr{C}$, provided with a suitable notion of cofibration, is a c-completion of $\mathscr{C}_{0}$. The definition of $\operatorname{Cof} \mathscr{C}$ is forced: a morphism in $\mathscr{C}$ is a cofibration if and only if it is a colimit of a cofibration in some $c\left(\Lambda, \mathscr{C}_{0}\right)$.

THEOREM 8.2. If $\mathscr{C}_{0}$ is a small c-category then the category $\mathscr{C}$ of c-accessible functors $\mathscr{C}_{0}^{\mathrm{op}} \rightarrow \mathscr{S}$ has the structure of a c-completion of $\mathscr{C}_{0}$.

We recall that in view of Theorem 7.4 this implies the existence of a unique c-completion for any small c-category.

All that is necessary here is to show that $(\mathscr{C}, \operatorname{Cof} \mathscr{C})$ satisfies axioms $\mathrm{C} 1,2$ for c-categories. As to axiom $\mathrm{Cl}$, it is clear that all morphisms $0 \rightarrow X$ and all isomorphisms are in $\operatorname{Cof} \mathscr{C}$. For the closure of $\operatorname{Cof} \mathscr{C}$ under composition we proceed as follows. 
Suppose $g: B^{\prime} \rightarrow B$ in $\operatorname{Cof} c\left(\Gamma, \mathscr{C}_{0}\right)$ and $h: A \rightarrow A^{\prime}$ in $\operatorname{Cof} c\left(\Lambda, \mathscr{C}_{0}\right)$, and that $w$ : colim $A \approx \operatorname{colim} B$. Then, exactly as in Lemma 5.2, we construct a realization $f: A p \rightarrow B j^{*}$ of $w$ in $c\left(\Delta, \mathscr{C}_{0}\right)$. In that category consider the diagram

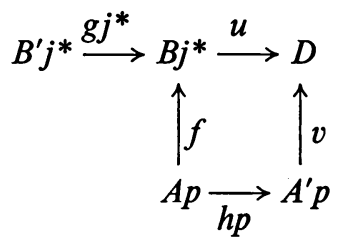

in which the square is a pushout. Then $u$ is a cofibration. Applying colim, which preserves pushouts, we see that $\operatorname{colim} v$, as a pushout of $w=\operatorname{colim} f$, is an isomorphism. Thus $(\operatorname{colim} h) w^{-1}(\operatorname{colim} g)=(\operatorname{colim} v)^{-1} \operatorname{colim}\left(u\left(g j^{*}\right)\right) \in \operatorname{Cof} \mathscr{C}$. But this is effectively the general case.

The pushout axiom C2 follows from a completely analogous argument.

9. Homotopy in $c(\Lambda, \mathscr{C})$. We suppose now that $\mathscr{C}$ is an h-c-category. If $\Lambda$ is an $\mathscr{L}$-lattice we shall introduce a homotopy relation in $c(\Lambda, \mathscr{C})$ in such a way as to make it too an h-c-category.

Let $\mathscr{E}$ be the class of morphisms $f$ of $c(\Lambda, \mathscr{C})$ such that for each $\lambda \in \Lambda, f_{\lambda}$ is a homotopy equivalence.

LEMMA 9.1. (i) $\mathscr{E}$ contains all identity morphisms; (ii) if any two of $f, g, f g$ are in $\mathscr{E}$ then so is the third; (iii) a pushout of a cofibration which is in $\mathscr{E}$ is again in $\mathscr{E}$.

This is more or less obvious. The following facts seem less so.

LEMMA 9.2. If $f: A \rightarrow B$ is a cofibration in $\mathscr{E}$ then $f$ has a left inverse.

We consider pairs $\left(\Lambda^{\prime}, g^{\prime}\right)$ where $\Lambda^{\prime}$ is an initial sublattice of and $g^{\prime}$ is a left inverse of the restriction of $f$ to $\Lambda^{\prime}$. If we order these by extension we may by Zorn's lemma conclude the existence of a maximal one; we now denote by $\left(\Lambda^{\prime}, g^{\prime}\right)$ such a maximal pair. We must show $\Lambda^{\prime}=\Lambda$.

If not, let $\gamma$ be minimal in $\Lambda-\Lambda^{\prime}$ and let $\lambda \in \Lambda^{\prime}$ be its immediate predecessor. Consider the diagram

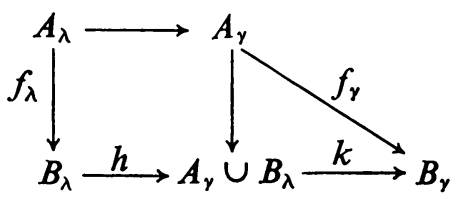

where the square is a pushout. Since $f_{\lambda}$ is a cofibration and a homotopy equivalence in $\mathscr{C}$ so also is $h$. Thus $k$ is a homotopy equivalence. But, since $f$ is a cofibration, $k$ is a cofibration too and thus has a left inverse, say $w$. Then $\left(1 g_{\gamma}\right) w: B_{\gamma} \rightarrow A_{\gamma}$ is a left inverse of $f_{\gamma}$. Since for any $\gamma^{\prime}$ in the sublattice generated by $\Lambda^{\prime}$ and $\gamma$ both 
$A_{\gamma^{\prime}}$ and $B_{\gamma^{\prime}}$ are pushouts, $g$ may be uniquely extended over that sublattice, contradicting the maximality of $\left(\Lambda^{\prime}, g^{\prime}\right)$.

LeMma 9.3. Any morphism $f: A \rightarrow B$ in $c(\Lambda, \mathscr{C})$ has a factorization $f=h$ where $g: A \rightarrow C$ is a cofibration and $h: C \rightarrow B$ is in $\mathscr{E}$.

We shall refer to such a factorization as an $\mathscr{E}$-mapping-cylinder.

As in the proof of 9.2 we proceed by induction on initial sublattices of $\Lambda$. We may suppose that $h g$ is such a factorization of the restriction of $f$ to an initial sublattice $\Lambda^{\prime}$, and that it is maximal with respect to further extension over $\Lambda$.

If $\Lambda^{\prime} \neq \Lambda$ take, as above, $\gamma$ minimal in $\Lambda-\Lambda^{\prime}$ and let $\lambda \in \Lambda^{\prime}$ be its maximal predecessor. If

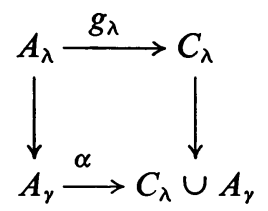

is a pushout then $\left(h_{\lambda} f_{\gamma}\right): C_{\lambda} \cup A_{\gamma} \rightarrow B_{\gamma}$ has a mapping cylinder factorization

$$
C_{\lambda} \cup A_{\gamma} \stackrel{\theta}{\longrightarrow} C_{\gamma} \stackrel{h_{\gamma}}{\longrightarrow} B_{\gamma} .
$$

We set $g_{\gamma}=\theta \alpha$. As in the proof of 9.2, $g$ and $h$ are then uniquely extendible to the sublattice generated by $\Lambda^{\prime}$ and $\gamma$. The $g_{\gamma^{\prime}}$, as pushouts, are cofibrations; the $h_{\gamma^{\prime}}$ are homotopy equivalences by 1.3 . We conclude thus that $\Lambda^{\prime}=\Lambda$.

If we define an $\mathscr{E}$-cylinder over $A \in c(\Lambda, \mathscr{C})$ to be a diagram

$$
A \vee A \stackrel{\left(i_{0} i_{1}\right)}{\longrightarrow} Z A \stackrel{\alpha}{\longrightarrow} A
$$

such that $\left(i_{0} i_{1}\right)$ is a cofibration, $\alpha \in \mathscr{E}$ and $\alpha\left(i_{0} i_{1}\right)=\left(\begin{array}{ll}1 & 1\end{array}\right)$, then 9.3 implies the existence of $\mathscr{E}$-cylinders.

LEMMA 9.4. If

$$
A \vee A \stackrel{\left(i_{0} i_{1}\right)}{\longrightarrow} Z A \stackrel{\alpha}{\longrightarrow} A \text { and } A \vee A \stackrel{\left(i_{0}^{\prime} i_{1}^{\prime}\right)}{\longrightarrow} Z^{\prime} A \stackrel{\alpha^{\prime}}{\longrightarrow} A
$$

are $\mathscr{E}$-cylinders then so are

$$
A \vee A \stackrel{\left(i_{1} i_{0}\right)}{\longrightarrow} Z A \stackrel{\alpha}{\longrightarrow} A \text { and } A \vee A \stackrel{\left(i_{0}^{\prime \prime} i_{2}^{\prime \prime}\right)}{\longrightarrow} Z A \oplus Z^{\prime} A \stackrel{\alpha^{\prime \prime}}{\longrightarrow} A
$$

where $Z A \oplus Z^{\prime} A$ is defined by the pushout diagram

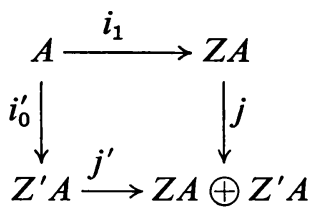

$i_{0}^{\prime \prime}=j i_{0}, i_{2}^{\prime \prime}=j^{\prime} i_{1}^{\prime}$ and $\alpha^{\prime \prime}=\left(\alpha \alpha^{\prime}\right)$. 
LEMMA 9.5. If

$$
A \vee A \stackrel{i}{\longrightarrow} Z A \stackrel{\alpha}{\longrightarrow} A \text { and } B \vee B \stackrel{j}{\longrightarrow} Z B \stackrel{\beta}{\longrightarrow} B
$$

are $\mathscr{E}$-cylinders and $f: A \rightarrow B$ then there is a $Z f: Z A \rightarrow Z B$ such that $(Z f) i=j(f \vee f)$.

Let

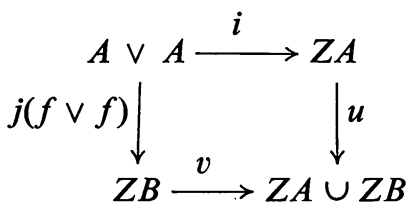

be a pushout, and let

$$
Z A \cup Z B \stackrel{k}{\longrightarrow} W \stackrel{\omega}{\longrightarrow} B
$$

be an $\mathscr{E}$-mapping-cylinder for $(f \alpha \beta)$. Then $\omega k v=\beta \in \mathscr{E}$ so that $k v$ is a cofibration in $\mathscr{E}$ and, by 9.2 , has a left inverse, say $\theta$. We need only set $Z f=\theta k u$.

We may now define a relation of homotopy between morphisms $f_{0}, f_{1}: A \rightarrow B$ in $c(\Lambda, \mathscr{C})$ by writing $f_{0} \simeq f_{1}$ if for some, and hence by 9.5 for any, $\mathscr{E}$-cylinder

$$
A \vee A \stackrel{\left(i_{0} i_{1}\right)}{\longrightarrow} Z A \longrightarrow A
$$

there is an $F: Z A \rightarrow B$ with $F i_{0}=f_{0}, f i_{1}^{\prime}=f_{1}$; we might refer to such an $F$ as an $\mathscr{E}$-homotopy of $f_{0}$ with $f_{1}$. Lemmas $9.4,9.5$ imply the following assertion.

LEMMA 9.6. The relation $\simeq$ is a congruence in $c(\Lambda, \mathscr{C})$.

What we are after, of course, is the following assertion.

Proposition 9.7. If $\mathscr{C}$ is an h-c-category then so is $c(\Lambda, \mathscr{C})$.

We begin by observing that any $f$ in $\mathscr{E}$ is a homotopy equivalence. Suppose first that $f$ is a cofibration and thus, by 9.2 , has a left inverse $g$. We shall show that $g \simeq 1_{B}$.

Let

$$
A \vee A \stackrel{i_{0} i_{1}}{\longrightarrow} Z A \stackrel{\alpha}{\longrightarrow} A
$$

be an $\mathscr{E}$-cylinder and let
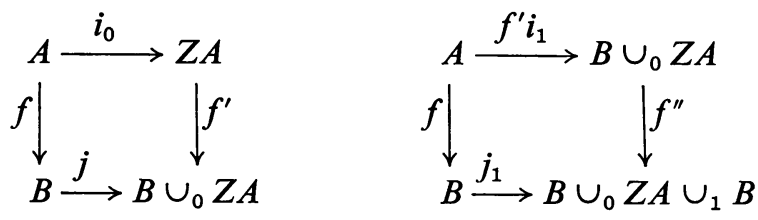
be pushouts. Then $j_{1}$ and $j_{0}=f^{\prime \prime} j$ are in $\mathscr{E}$. But $\left(j_{0} j_{1}\right)$ is also the pushout of $\left(i_{0} i_{1}\right)$ by $f \vee f$ and is thus a cofibration, and

$$
B \vee B \stackrel{\left(j_{0} j_{1}\right)}{\longrightarrow} B \cup_{0} Z A \cup_{1} B \stackrel{(1 f \alpha 1)}{\longrightarrow} B
$$

is accordingly an $\mathscr{E}$-cylinder. The $\mathscr{E}$-homotopy in question is

$$
(1 f \alpha f g): B \cup_{0} Z A \cup \cup_{1} B \rightarrow B \text {. }
$$

For the general case we need only construct (9.3) an $\mathscr{E}$-mapping-cylinder

$$
A \vee B \stackrel{(i j)}{\longrightarrow} W \longrightarrow B
$$

for $(f 1)$ and apply our observations to $i$ and $j$.

But now axiom HC3 follows at once from 9.1, while 9.3 becomes HC4. Axiom $\mathrm{HCl}$ is immediate, since if $A \vee A \rightarrow Z A \rightarrow A$ and $B \vee B \rightarrow Z B \rightarrow B$ are $\mathscr{E}$-cylinders (we might as well by now just say "cylinders") then so is

$$
A \vee B \vee A \vee B \rightarrow Z A \vee Z B \rightarrow A \vee B
$$

Finally, for HC2, suppose $f: A \rightarrow B$ is a cofibration and $A \vee A \rightarrow Z A \rightarrow A$ is a cylinder. Then (as in the proof of 1.2) we may construct a cylinder $B \vee B \rightarrow Z B \rightarrow B$ and a morphism $Z f: Z A \rightarrow Z B$ such that

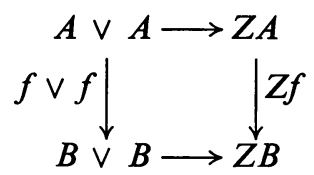

is a 2-cofibration. The proof of homotopy extension then proceeds in the manner familiar in topology.

The following observation requires no additional proof.

Proposition 9.8. If $\Lambda, \Gamma$ are in $\mathscr{L}$ and $\varphi: \Lambda \rightarrow \Gamma$ is a lattice map, and if $F: \mathscr{C} \rightarrow \mathscr{D}$ is an h-c-functor then the functor $c(\Gamma, \mathscr{C}) \rightarrow c(\Lambda, \mathscr{D})$ induced by composition is an h-c-functor.

10. Homotopy in c-completions. Let $\mathscr{C}_{0}$ be a small h-c-category. If we forget the homotopy relation $\mathscr{C}_{0}$ has a c-completion $\mathscr{C}$. We propose to extend the homotopy congruence to $\mathscr{C}$ in such a way that it too becomes an h-c-category.

LEMmA 10.1. If $\Lambda, \Gamma \in \mathscr{L}$ and $A \vee A \rightarrow Z A, B \vee B \rightarrow Z B$ are cylinders in $c\left(\Lambda, \mathscr{C}_{0}\right)$, $c\left(\Gamma, \mathscr{C}_{0}\right)$ then for any $f: \operatorname{colim} A \rightarrow \operatorname{colim} B$ in $\mathscr{C}$ there is a $Z f: \operatorname{colim} Z A \rightarrow \operatorname{colim} Z B$ such that 


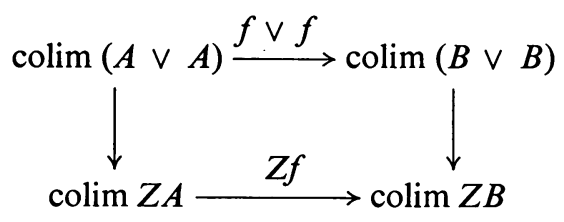

commutes.

This follows easily from 5.2.

We now proceed to define the homotopy relation in $\mathscr{C}$. We say that $f_{0} \simeq f_{1}: X \rightarrow Y$ in $\mathscr{C}$ if, for some $\Lambda$, there is a cylinder

$$
A \vee A \stackrel{\left(i_{0} i_{1}\right)}{\longrightarrow} Z A
$$

in $c\left(\Lambda, \mathscr{C}_{0}\right)$ with $X=\operatorname{colim} A$ and an $F: \operatorname{colim} Z A \rightarrow Y$ such that $F$ colim $i_{0}=f_{0}$, $F$ colim $i_{1}=f_{1}$. It follows from 10.1 that there must be such an $F$ for any such cylinder, and we may in routine fashion verify the following statement.

LEMMA 10.2. The homotopy relation $\simeq$ is a congruence in $\mathscr{C}$. Two morphisms in $\mathscr{C}$ are homotopic if and only if they are colimits of a pair of homotopic morphisms in some $c\left(\Lambda, \mathscr{C}_{0}\right)$.

The central result in this connection is of course the following.

THEOREM 10.3. If $\mathscr{C}_{0}$ is a small h-c-category and $\mathscr{C}$ is its c-completion then $\mathscr{C}$ has the structure of an h-c-category with each of the functors colim: $c\left(\Lambda, \mathscr{C}_{0}\right) \rightarrow \mathscr{C}$ an h-c-functor.

Axioms $\mathrm{HCl}$ and $\mathrm{HC} 4$ are obviously satisfied. For $\mathrm{HC} 2$ we observe that 10.2 and 5.2 allow us to reduce the homotopy extension problem in $\mathscr{C}$ to a corresponding one in some $c\left(\Lambda, \mathscr{C}_{0}\right)$.

For HC3 we proceed in the following manner. Suppose $a: A^{\prime} \rightarrow A$ is a cofibration in $c\left(\Lambda, \mathscr{C}_{0}\right)$ such that colim $a$ is a homotopy equivalence in $\mathscr{C}$. Since HC2 holds in $\mathscr{C}$, colim $a$ has a left inverse. Applying 5.2, we conclude the existence of a cofinal lattice map $p: \Gamma \rightarrow \Lambda$ and a diagram

$$
A^{\prime} p \stackrel{a p}{\longrightarrow} A p \stackrel{r}{\longrightarrow} \bar{A}
$$

in $c\left(\Gamma, \mathscr{C}_{0}\right)$ such that colim $\bar{A}=$ colim $A^{\prime} p$ and colim $r$ is a left inverse of colim ap.

Now if

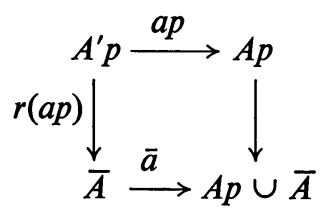

is a pushout in $c(\Gamma, \mathscr{C})$ then colim $\bar{a}$ is isomorphic in $c([2], \mathscr{C})$ to colim $a$. But $\bar{a}$ has a left inverse in $c\left(\Gamma, \mathscr{C}_{0}\right)$, namely $(r 1)$. 
Thus we might just as well start with a cofibration $a$ in $c\left(\Lambda, \mathscr{C}_{0}\right)$ having a left inverse $r$, and such that colim $a$ is a homotopy equivalence $r$. Now let

$$
A \stackrel{\tilde{r}}{\longrightarrow} \tilde{A} \stackrel{r^{\prime}}{\longrightarrow} A^{\prime}
$$

be a mapping cylinder in $c\left(\Lambda, \mathscr{C}_{0}\right)$ for $r$. Then $r^{\prime}$ is a homotopy equivalence in $c\left(\Lambda, \mathscr{C}_{0}\right)$ and $\tilde{r} a$, as a right inverse of $r^{\prime}$, is also a homotopy equivalence.

Since all of this is preserved in passing to $c\left(\Gamma, \mathscr{C}_{0}\right)$ by a cofinal lattice map $\Gamma \rightarrow \Lambda$, it is sufficient to consider a pushout of $a$ along a morphism $f: A^{\prime} \rightarrow B$ in $c\left(\Lambda, \mathscr{C}_{0}\right)$. We consider the diagram

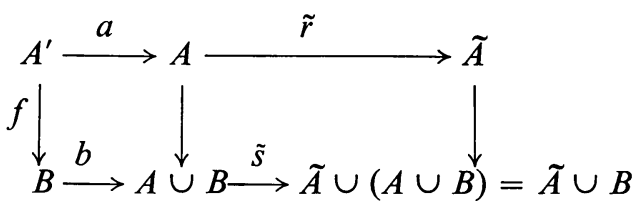

in which both squares are pushouts. Since the rectangle is also a pushout, $\tilde{s} b$ and hence colim $\tilde{s} b$ are homotopy equivalences.

Now colim $a$ and colim $\tilde{r}$ both have left inverses. Thus, as their pushouts, colim $b$ and colim $\tilde{s}$ have left inverses, say $t$ and $\tilde{t}$. But $t \tilde{t}$ is a left inverse of colim $(\tilde{s} b)$ and is thus a homotopy inverse. We conclude that $(\operatorname{colim} \tilde{s})(\operatorname{colim} b) t \tilde{t} \simeq 1_{\tilde{A} \cup B}$, so that $(\operatorname{colim} b) t \simeq \tilde{t} \operatorname{colim} \tilde{s}=1_{B \cup A}$ and $t$ is a homotopy inverse of colim $b$.

11. The Whitehead condition. The following statement is an easy consequence of 7.2.

Proposition 11.1. If $\mathscr{C}$ is the c-completion of the small h-c-category $\mathscr{C}_{0}, A \in \mathscr{C}_{0}$ and $X \in c(\Lambda, \mathscr{C})$ then colim $\mathscr{C} \square(A, X) \approx \mathscr{C} \square(A$, colim $X)$.

For $A$ has a cylinder

$$
A \vee A \stackrel{\left(i_{0} i_{1}\right)}{\longrightarrow} Z A
$$

in $\mathscr{C}_{0}$ and $\mathscr{C} \square(A, W)$ is the coequalizer of $\mathscr{C}(Z A, W) \rightarrow \mathscr{C}(A, W)$, induced by $i_{0}$ and $i_{1}$.

In the other direction we need a condition which must pass in this context for the well-known theorem of J. H. C. Whitehead. To facilitate its statement we say that a class $\mathscr{W}$ of objects (or a full subcategory) in a category $\mathscr{A}$ is a Whitehead class (subcategory) if for any $f: X \rightarrow Y$ in $\mathscr{A}, \mathscr{A}(W, X) \approx \mathscr{A}(W, Y)$ for all $W \in \mathscr{W}$ implies $f: X \approx Y$. If we observe that, $\mathscr{C}_{0}$ being a small h-c-category, $\mathscr{C}_{0}^{\square}$ is a full subcategory of $\mathscr{C}^{\square}$, we say that $\mathscr{C}_{0}$ is connective if $\mathscr{C}_{0}^{\square}$ is a Whitehead subcategory of $\mathscr{C}^{\square}$.

An example or two is in order. If $\mathscr{C}_{0}$ is the category of pointed finite connected $\mathrm{CW}$-complexes, so that $\mathscr{C}$ is the category of pointed connected $\mathrm{CW}$-complexes, then $\mathscr{C}_{0}$ is connective. This follows immediately from the usual Whitehead theorem 
which says in fact that the spheres form a Whitehead class in $\mathscr{C}^{\square}$. On the other hand if $\mathscr{C}_{0}$ is the category of all pointed finite $\mathrm{CW}$-complexes then $\mathscr{C}_{0}$ is not connective. This may be seen as follows. Let $\pi$ be the group of all permutations of $Z$ with finite support, and let $\pi^{\prime}$ be the subgroup leaving 0 fixed. If $i: \pi^{\prime} \subset \pi$ is the inclusion then $K(i, 1)^{+}: K\left(\pi^{\prime}, 1\right)^{+} \rightarrow K(\pi, 1)^{+}$induces bijections $\mathscr{C} \square\left(A, K\left(\pi^{\prime}, 1\right)^{+}\right)$ $\approx \mathscr{C}^{\square}\left(A, K(\pi, 1)^{+}\right)$for all $A \in \mathscr{C}_{0}$.

It is not clear how to characterize the condition that $\mathscr{C}_{0}$ be connective in "internal" terms (but see $\$ 13$ below). What survives of the Whitehead theorem is the following lemma.

LeMmA 11.2. If $\mathscr{C}$ is the c-completion of the small h-c-category $\mathscr{C}_{0}, Y \in \mathscr{C}$ and $\mathscr{C}^{\square}(A, Y)=0$, all $A \in \mathscr{C}_{0}$ then $Y$ is contractible.

It is enough to show that for any cofibration $X^{\prime} \rightarrow X$ in $\mathscr{C}, \mathscr{C}(X, Y) \rightarrow \mathscr{C}\left(X^{\prime}, Y\right)$ is surjective. This is done by a transfinite induction over initial sublattices in the manner employed passim above.

When $\mathscr{C}_{0}$ is connective we may make the following observations.

Proposition 11.3. If $\mathscr{C}$ is the c-completion of the small connective h-c-category $\mathscr{C}_{0}$ then each of the functors colim: $c(\Lambda, \mathscr{C}) \rightarrow \mathscr{C}$ is an h-c-functor.

We know by 7.2 that each of the colimit functors is a c-functor. But they also preserve homotopy equivalences and hence the homotopy relation.

In particular a coproduct of cylinders is a cylinder over the coproduct, which implies the following statement.

Proposition 11.4. The canonical functor $\mathscr{C} \rightarrow \mathscr{C}^{\square}$ preserves coproducts.

This is, perhaps, the place to introduce the notion of a translimit. If $\mathscr{D}$ is a small category and $\mathscr{A}_{0} \subset \mathscr{A}$ is a full subcategory a translimit of a functor $F: \mathscr{D} \rightarrow \mathscr{A}$ (with respect to $\mathscr{A}_{0}$ ) is an object $A \in \mathscr{A}$, together with morphisms $\mathscr{A}_{D}: F D \rightarrow A$ for $D \in \mathscr{D}$, such that for $d: D^{\prime} \rightarrow D$ in $\mathscr{D}, \mathscr{A}_{D}\left(F d^{\prime}\right)=\mathscr{A}_{D^{\prime}}$, constituting a weak colimit of $F$, i.e., such that any morphism $\varphi$ of $F$ into a constant functor factors (not necessarily uniquely) through $A$, and such that for all $B \in \mathscr{A}_{0}$, colim $\mathscr{A}(B, F)$ $\approx \mathscr{A}(B, A)$.

Lemma 11.5. If $\mathscr{A}_{0}$ is a Whitehead subcategory of $\mathscr{A}$ then any two translimits of $F: \mathscr{D} \rightarrow \mathscr{A}$ are isomorphic.

The isomorphism need not, of course, be canonical. Compare the notion of injective envelope.

The example we have in mind is the following. Suppose that $\mathscr{C}$ is the c-completion of $\mathscr{C}_{0}$ and that $X: \Lambda \rightarrow \mathscr{C}$ is a c-system. If $\eta: \mathscr{C} \rightarrow \mathscr{C} \square$ is the canonical functor then $\eta$ colim $X$ is a translimit of $\eta X$. On the other hand it is far from clear when a functor $F: \Lambda \rightarrow \mathscr{C} \square$ possesses a translimit. This question is closely related to the 
missing part of Brown's theorem (see below, \$12). We can however make the following assertion.

Proposition 11.6. If $N=\{0,1,2, \ldots\}$ then any functor $F: N \rightarrow \mathscr{C}^{\square}$ has a translimit.

For any such functor may be factored as $\eta X$ with $X: N \rightarrow \mathscr{C}$ a c-system.

12. Brown's representation theorem. The following theorem is due to $E$. $H$. Brown [2]. It is of especial interest to us because it seems especially applicable to the homotopy category of a c-completion. We include the proof here as being very much in the context of our discussion.

THEOREM 12.1. Let $\mathscr{A}$ be a pointed category and $\mathscr{A}_{0}$ a full subcategory such that (i) $\mathscr{A}$ has coproducts and weak pushouts, (ii) $\mathscr{A}_{0}$ is a small Whitehead subcategory, (iii) every sequence in $\mathscr{A}$ has a translimit with respect to $\mathscr{A}_{0}$. Then a functor $\mathscr{A}^{\mathrm{op}} \rightarrow \mathscr{S}$ is representable if and only if it preserves products and weak pullbacks.

Observe that these conditions mean that coproducts and weak pushouts in $\mathscr{A}$ go, respectively, into products and weak pullbacks in $\mathscr{S}$.

We remark also that $\mathscr{A}$ is provided with weak coequalizers: if $f_{0}, f_{1}: A^{\prime} \rightarrow A$ and

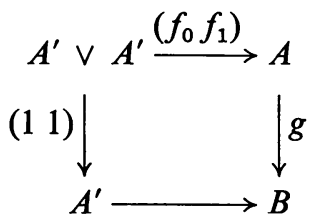

is a weak pushout then $g$ is a weak coequalizer of $f_{0}$ and $f_{1}$.

The necessity of the two conditions for representability needs no discussion. Let us suppose that $F: \mathscr{A}^{\text {op }} \rightarrow \mathscr{S}$ satisfies them. Suppose also that $\mathscr{A}_{1}$ is a small full subcategory of $\mathscr{A}$ containing $\mathscr{A}_{0}$. We shall demonstrate the following statement.

$\left(^{*}\right)$ There is an $X \in \mathscr{A}$ and a morphism $\alpha: \mathscr{A}(-, X) \rightarrow F$ such that $\alpha_{A}$ is surjective for $A \in \mathscr{A}_{1}$ and bijective for $A \in \mathscr{A}_{0}$.

Since $F$ is product preserving it follows from the Yoneda lemma that there is an $X_{0} \in \mathscr{A}$ and an $\alpha_{0}: \mathscr{A}\left(-, X_{0}\right) \rightarrow F$ with $\alpha_{0, A}$ surjective for $A \in \mathscr{A}_{1}$ : for example, take

$$
X_{0}=\bigvee_{A \in \mathscr{A}_{1}, u \in F A} A
$$

Moreover if $R$ is the equivalence relation on $\mathscr{A}\left(-, X_{0}\right)$ induced by $\alpha_{0}$ then $R$ is again product preserving, so that there is a $Y_{0} \in \mathscr{A}$ and $u_{0}^{\prime}, u_{0}^{\prime \prime}: Y_{0} \rightarrow X_{0}$ such that

$$
\mathscr{A}\left(A, Y_{0}\right) \rightarrow \mathscr{A}\left(A, X_{0}\right) \rightarrow F A
$$

is a coequalizer diagram for any $A \in \mathscr{A}_{1}$.

If

$$
X_{0} \stackrel{v_{0}}{\longrightarrow} X_{1}
$$


is a weak coequalizer of $u_{0}^{\prime}, u_{0}^{\prime \prime}$ then, since $F$ preserves weak pullbacks, there is an $\alpha_{1}: \mathscr{A}\left(-, X_{1}\right) \rightarrow F$ such that $\alpha_{0}=\alpha_{1} \mathscr{A}\left(-, v_{0}\right)$. Clearly $\alpha_{1, A}$ is still surjective for $A \in \mathscr{A}_{1}$.

Iterating this construction we get a sequence

$$
X_{0} \stackrel{v_{0}}{\longrightarrow} X_{1} \stackrel{v_{1}}{\longrightarrow} X_{2} \longrightarrow \cdots,
$$

morphisms $u_{0}^{\prime}, u_{0}^{\prime \prime}: Y_{i} \rightarrow X_{i}$ and a morphisms of functors $\alpha_{i}: \mathscr{A}\left(-, X_{i}\right) \rightarrow F$ such that the diagrams

$$
\begin{aligned}
& \mathscr{A}\left(-, Y_{i}\right) \longrightarrow \mathscr{A}\left(-, X_{i}\right) \stackrel{\alpha_{i}}{\longrightarrow} F
\end{aligned}
$$

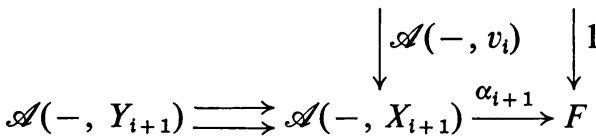

all commute, and such that the rows are coequalizer diagrams for arguments $A \in \mathscr{A}_{1}$. From this we get in turn a morphism $\bar{\alpha}$ : colim $\mathscr{A}\left(-, X_{i}\right) \rightarrow F$ such that $\bar{\alpha}_{A}$ is bijective for $A \in \mathscr{A}_{1}$. If $X$ is a translimit of $X_{0} \rightarrow X_{1} \rightarrow \cdots$ then $\bar{\alpha}$ factors through $\alpha: \mathscr{A}(-, X) \rightarrow F$ which is easily seen to have the required properties.

But the theorem follows almost immediately. For suppose $X$ and $\alpha: \mathscr{A}(-, X) \rightarrow F$ are the object and morphism given by $\left({ }^{*}\right)$. Then, applying $\left(^{*}\right)$ once more, after adding $X$ to $\mathscr{A}_{1}$, we conclude the existence of an $X^{\prime}$ and an $\alpha^{\prime}: \mathscr{A}\left(-, X^{\prime}\right) \rightarrow F$ which is surjective on $X$ as well. If $\alpha_{X} 1_{X}=\alpha_{X}^{\prime} f$ then $f: X \rightarrow X^{\prime}$ and $\mathscr{A}(A, f)$ : $\mathscr{A}(A, X) \approx \mathscr{A}(A, X)$ for all $A \in \mathscr{A}_{0}$. Since $\mathscr{A}_{0}$ is a Whitehead subcategory, $f$ is an isomorphism. But $\mathscr{A}_{1}$ was arbitrary; we conclude that $\alpha_{W}$ is surjective for all $W \in \mathscr{A}$.

It remains only to show that all $\alpha_{W}$ are injective as well. Suppose $f_{0}, f_{1}: W \rightarrow X$ and $\alpha_{W} f_{0}=\alpha_{W} f_{1}$, which is to say that $\left(F f_{0}\right) \alpha_{X} 1_{X}=\left(F f_{1}\right) \alpha_{X} 1_{X}$. If $g: X \rightarrow Y$ is a weak coequalizer of $f_{0}$ and $f_{1}$ we may conclude that $\alpha_{X} 1_{X}=(F g) \alpha_{Y} h=F(h g) \alpha_{X} 1_{X}$ for some $h: Y \rightarrow X$. But then for $A \in \mathscr{A}_{0}, \mathscr{A}(A, h g)$ is bijective so that $h g$ is an isomorphism. Since $h g f_{0}=h g f_{1}$ we conclude that $f_{0}=f_{1}$.

The point of this theorem, in the present context, is that if $\mathscr{C}$ is the c-completion of the small connective h-c-category $\mathscr{C}_{0}$ then the pair $\mathscr{C}^{\square}, \mathscr{C}_{0}^{\square}$ satisfies the hypotheses of 12.1. Condition (i) follows from 11.4 and the observation that a c-pushout in $\mathscr{C}$ goes into a weak pushout in $\mathscr{C}^{\square}$; condition (ii) is the Whitehead theorem; condition (iii) is 11.6. Furthermore it is easy to see that a functor $F: \mathscr{C}^{\square \mathrm{op}} \rightarrow \mathscr{S}$ takes weak pushouts into weak pullbacks if and only if $F_{\eta}: \mathscr{C}^{\mathrm{op}} \rightarrow \mathscr{S}$ takes c-pushouts into weak pullbacks. Such a functor $F \eta$ is said to be half-exact (cf. Dold [3]).

THEOREM 12.2. If $\mathscr{C}$ is the c-completion of a small connective h-c-category $\mathscr{C}_{0}$ then a functor $F: \mathscr{C}^{\square \mathrm{op}} \rightarrow \mathscr{S}$ is representable if and only if $F_{\eta}$ is half-exact and takes coproducts into products.

The following corollaries are immediate. 
COROLlaRY 12.3. $\mathscr{C}^{\square}$ is closed under products.

COROLlaRY 12.4. If $\mathscr{C}_{0}, \mathscr{C}_{0}^{\prime}$ are small connective h-c-categories and $F_{0}: \mathscr{C}_{0} \rightarrow \mathscr{C}_{0}^{\prime}$ is an h-c-functor with c-continuous extension $F: \mathscr{C} \rightarrow \mathscr{C}^{\prime}$ to the c-completions then $F \square: \mathscr{C}^{\square} \rightarrow \mathscr{C}^{\prime \square}$ has an adjoint.

By applying the Whitehead condition to the adjunction morphisms, we get the following further corollary.

COROLlaRY 12.5. If, under the hypotheses of $12.4, F_{0}^{\square}: \mathscr{C}_{0}^{\square} \rightarrow \mathscr{C}_{0}^{\prime \square}$ is an equivalence of categories then so also is $F^{\square:} \mathscr{C}^{\square} \rightarrow \mathscr{C}^{\prime} \square$.

There is a complement to Brown's theorem which asserts that if $\mathscr{C}_{0}^{\square}$ is countable and $F: \mathscr{C}_{0}^{\text {op }} \rightarrow \mathscr{S}$ is half-exact and has its values in the category of countable sets then $F$ extends uniquely to a product-preserving half-exact functor $\mathscr{C}^{\text {op }} \rightarrow \mathscr{S}$, which is accordingly representable at the homotopy level. The requirement of countability is somewhat mysterious here, but no improvement of the hypotheses seems to be known. Without attempting to contribute to the solution of this problem, we might nevertheless remark that it seems to be connected with the existence of nonsequential translimits $\left({ }^{5}\right)$.

13. Stabilization. We use the word "stable" in two ways. If $\Psi: \mathscr{A} \rightarrow \mathscr{A}$ and $\Psi^{\prime}: \mathscr{A}^{\prime} \rightarrow \mathscr{A}^{\prime}$ are functors we say that a functor $F: \mathscr{A} \rightarrow \mathscr{A}^{\prime}$ is stable (with respect to $\left.\Psi, \Psi^{\prime}\right)$ if $\Psi^{\prime} F=F \Psi$. If $F, G: \mathscr{A} \rightarrow \mathscr{A}^{\prime}$ are stable functors, a morphism $\varphi: F \rightarrow G$ is stable if $\varphi \Sigma=\Sigma \varphi$, i.e., $\varphi_{\Sigma A}=\Sigma \varphi_{A}$ for all $A \in \mathscr{A}$. We say that the category $\mathscr{A}$ is stable (with respect to $\Psi$ ) when $\Psi$ is an automorphism.

The colimit $\operatorname{Stab}_{\Psi} \mathscr{A}$ of the sequence

$$
\stackrel{\Psi}{\longrightarrow} \mathscr{A}_{-1} \stackrel{\Psi}{\longrightarrow} \mathscr{A}_{0} \stackrel{\Psi}{\longrightarrow} \mathscr{A}_{1} \stackrel{\Psi}{\longrightarrow} \cdots
$$

in which each $\mathscr{A}_{i}$ in $\mathscr{A}$, is the stabilization of $\mathscr{A}$ (with respect to $\Psi$ ). A functor $\operatorname{Stab}_{\Psi} \mathscr{A} \rightarrow \operatorname{Stab}_{\Psi} \mathscr{A}$, again denoted by $\Psi$, is defined by $\Psi \operatorname{inj}_{q}=\operatorname{inj}_{q-1}$; it is clearly an automorphism so that $\operatorname{Stab}_{\Psi} \mathscr{A}$ is stable. The functor $\psi=\operatorname{inj}_{0}: \mathscr{A} \rightarrow \operatorname{Stab}_{\Psi} \mathscr{A}$ is stable and universal for stable functors into stable categories, in the sense that any such functor $F$ factors uniquely as $F^{\prime} \psi$ with $F^{\prime}$ stable.

If $F: \mathscr{A} \rightarrow \mathscr{A}^{\prime}$ is stable (with respect to $\Psi, \Psi^{\prime \prime}$ ) it induces a stable functor Stab $F: \operatorname{Stab}_{\Psi} \mathscr{A}^{\prime} \rightarrow \operatorname{Stab}_{\Psi} \mathscr{A}^{\prime}$ by (Stab $\left.F\right) \psi=\psi^{\prime} F$; we shall usually allow ourselves to write $F$ instead of Stab $F$. If $\varphi: F \rightarrow G$ is a stable morphism it induces similarly a morphism Stab $\varphi:$ Stab $F \rightarrow \operatorname{Stab} G$.

Stabilization is of course functorial. In particular if $\varphi$ is an isomorphism then so is $\operatorname{Stab} \varphi$.

${ }^{5}$ ) Added in proof. J. F. Adams has recently succeeded in removing the countability hypothesis in the stable case (personal communication). 
If $F: \mathscr{A} \rightarrow \mathscr{A}^{\prime}$, instead of being stable, satisfies the weaker condition $F \Psi \approx \Psi^{\prime} F$ we say it is weakly stable. A category $\mathscr{A}$ is weakly stable (with respect to $\Psi: \mathscr{A} \rightarrow \mathscr{A}$ ) when $\Psi$ is an equivalence of $\mathscr{A}$ with itself. When "stable" is replaced by "weakly stable" the universal factorization property remarked above holds up to isomorphism instead of uniquely. We may make, moreover, the following observation.

Proposition 13.1. If $\mathscr{A}$ is weakly stable (with respect to $\Psi$ ) then $\Psi: \mathscr{A} \rightarrow \operatorname{Stab}_{\Psi} \mathscr{A}$ is an equivalence of categories.

If $\mathscr{A}$ is provided with two commuting endofunctors, i.e., with two functors each of which is stable with respect to the other, say $\Phi, \Psi: \mathscr{A} \rightarrow \mathscr{A}$, these induce functors $\Phi: \operatorname{Stab}_{\Psi} \mathscr{A} \rightarrow \operatorname{Stab}_{\Psi} \mathscr{A}$ and $\Psi: \operatorname{Stab}_{\Phi} \mathscr{A} \rightarrow \operatorname{Stab}_{\Phi} \mathscr{A}$. The resulting double stabilizations $\operatorname{Stab}_{\Phi} \operatorname{Stab}_{\Psi} \mathscr{A}, \operatorname{Stab}_{\Psi} \operatorname{Stab}_{\Phi} \mathscr{A}$ may be identified with the colimit $\mathrm{Stab}_{\Phi, \Psi} \mathscr{A}$ of the diagram

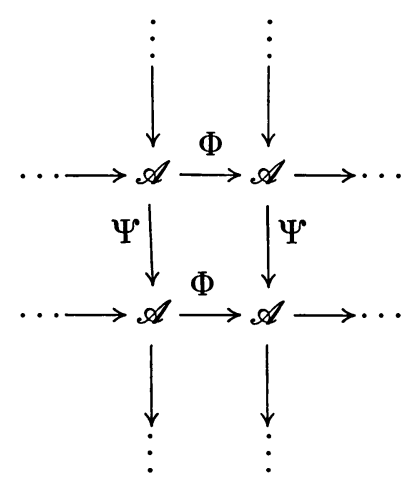

If a category is provided with some additional structure, for example that of a c-category or an h-c-category, we shall generally confine our attention to endofunctors preserving this structure.

Proposition 13.2. If $\mathscr{C}$ is a c-category and $\Psi: \mathscr{C} \rightarrow \mathscr{C}$ is a c-functor then $\operatorname{Stab}_{\Psi} \mathscr{C}$ may be given the structure of a stable c-category in such a way that $\psi: \mathscr{C} \rightarrow \mathrm{Stab}_{\Psi} \mathscr{C}$ is universal for stable c-functors into stable c-categories.

For any morphism in $\operatorname{Stab}_{\Psi} \mathscr{C}$ may be written as $\Psi^{a} \psi f$ for some $f$ in $\mathscr{C}$ and some $q=0, \pm 1 . \pm 2, \ldots$; we take as cofibrations those for which $\Psi^{n} f$ is a cofibration for large $n$.

Proposition 13.3. If further $\mathscr{C}$ is an h-c-category and $\Psi$ an h-c-functor then $\mathrm{Stab}_{\Psi} \mathscr{C}$ becomes an h-c-category with $\psi$ universal for stable h-c-functors into stable h-c-categories.

Any pair of morphisms with the same domain and codomain in $\mathrm{Stab}_{\Psi} \mathscr{C}$ may be written as $\Psi^{q} \psi f, \Psi^{q} \psi g$ where $f, g$ have the same domain and codomain in $\mathscr{C}$. We call them homotopic if $\Psi^{n} f \simeq \Psi^{n} g$ for large $n$. 
Under the hypotheses of $13.2, \Psi$ induces a functor $\Psi^{\square}: \mathscr{C}^{\square} \rightarrow \mathscr{C}^{\square}$. The universal property of the stabilization provides a functor

$$
\operatorname{Stab}_{\Psi \square} \mathscr{C}^{\square} \rightarrow\left(\operatorname{Stab}_{\Psi} \mathscr{C}\right)^{\square} .
$$

Proposition 13.4. Under the hypotheses of 13.2,

$$
\operatorname{Stab}_{\Psi} \square \mathscr{C}^{\square} \approx\left(\operatorname{Stab}_{\Psi} \mathscr{C}\right)^{\square} .
$$

The case of greatest interest for us is the following. If $\mathscr{C}$ is an h-c-category then $\mathscr{C}^{\square}$ is provided with a functor $\Sigma: \mathscr{C}^{\square} \rightarrow \mathscr{C}^{\square}$, the suspension, defined uniquely up to canonical isomorphism by the existence of cofibrations $X \rightarrow C X$ in $\mathscr{C}$ with $C X$ contractible, having $\Sigma X$ as cofiber. We shall say that $\Psi: \mathscr{C} \rightarrow \mathscr{C}$ is a suspension functor (or a lifted suspension functor) in $\mathscr{C}$ if it is an h-c-functor such that $\Psi^{\square}=\Sigma$. In this case we shall allow ourselves to write $\Sigma$ for $\Psi$.

The category $\operatorname{Stab}_{\Sigma} \mathscr{C} \square$ is always supplied with a "triangulation" in the sense of Puppe ([8], also [6]) which we may denote by $\Delta_{\text {cof }}$ and refer to as the "cofibration triangulation". The category $\left(\operatorname{Stab}_{\Sigma} \mathscr{C}\right)^{\square}$, being already stable, is also supplied with a cofibration triangulation. It is not our intention to expand further on this structure, or even to give the precise definitions, here. But we record the fact that these two triangulations correspond-of course-under the isomorphism of 13.3.

We have seen that stabilization commutes with passage to homotopy. It does not, on the other hand, commute with c-completion; this is why a theory of c-completions is necessary even in the "classical" case of CW-complexes.

The utility of such a theory is enhanced by the following "stable Whitehead theorem".

Proposition 13.5. A small h-c-category which is stable with respect to some lifted suspension functor is connective.

For the homotopy category of its c-completion is triangulable; the conclusion follows at once from 11.2.

Let $\mathscr{C}_{0}$ be a small h-c-category and $\mathscr{C}$ its c-completion. If $\Sigma$ is a lifted suspension functor for $\mathscr{C}_{0}$ then $\mathrm{Stab}_{\Sigma} \mathscr{C}_{0}$ is again a small h-c-category. We denote the ccompletion of $\operatorname{Stab}_{\Sigma} \mathscr{C}_{0}$ by $\mathscr{B}_{\Sigma} \mathscr{C}_{0}$; this is the Boardman completion of $\mathscr{C}_{0}$. In view of 7.3 and the universal property of the stabilization there is a unique stable functor $\operatorname{Stab}_{\Sigma} \mathscr{C} \rightarrow \mathscr{B}_{\Sigma} \mathscr{C}_{0}$ such that the composition $\mathscr{C} \rightarrow \operatorname{Stab}_{\Sigma} \mathscr{C} \rightarrow \mathscr{B}_{\Sigma} \mathscr{C}_{0}$ is a c-continuous extension of $\mathscr{C}_{0} \rightarrow \mathscr{B}_{\Sigma} \mathscr{C}_{0}$. This functor is not in general an equivalence of categories; indeed the induced functor $\left(\operatorname{Stab}_{\Sigma} \mathscr{C}\right)^{\square} \rightarrow\left(\mathscr{B}_{\Sigma} \mathscr{C}_{0}\right)^{\square}$ also fails to be an equivalence.

For example if $\mathscr{C}_{0}$ is the category of finite $\mathrm{CW}$-complexes, so that $\mathscr{C}$ is the category of all CW-complexes, it is easy to see that the family $\left\{\Sigma^{n} S^{0} \mid n \in Z\right\}$ has no coproduct in $\operatorname{Stab}_{\Sigma} \mathscr{C}$, nor even in $\left(\operatorname{Stab}_{\Sigma} \mathscr{C}\right) \square$. As a matter of fact it is an amusing exercise to show that the subfamily $\left\{\Sigma^{n} S^{0} \mid n \geqq 0\right\}$ lacks a coproduct in $\left(\operatorname{Stab}_{\Sigma} \mathscr{C}\right)^{\square}$. 
For any small h-c-category $\mathscr{C}_{0}$ with a lifted suspension $\Sigma$, on the other hand, $\mathscr{B}_{\Sigma} \mathscr{C}_{0}$ is c-complete. Its homotopy category is triangulated and closed under products and coproducts. It follows immediately from the axioms for triangulations that products and coproducts of cofibration triangles are again cofibration triangles.

Further, as a triangulated category with coproducts, $\left(\mathscr{B}_{\Sigma} \mathscr{C}_{0}\right)^{\square}$ embeds as the full subcategory of injectives in a Frobenius category $\mathscr{H}_{B_{\Sigma}} \mathscr{C}_{0}$ ([5], also [6]), which must accordingly be both complete and cocomplete.

As our notation indicates, $\mathscr{B}_{\Sigma} \mathscr{C}_{0}$ depends strongly on the lifted suspension functor $\Sigma$. This dependence is somewhat mitigated by the fact that in many examples (e.g. topological spaces, CW-complexes) there is a "natural" choice for $\Sigma$. Further, 12.5 gives some ground for hoping that the dependence of $\left(\mathscr{B}_{\Sigma} \mathscr{C}_{0}\right)^{\square}$ on $\Sigma$ is less strong. Unfortunately we have no general theorem to this effect.

14. Smash pairings and smash products. If $\mathscr{C}, \mathscr{C}^{\prime}, \mathscr{C}^{\prime \prime}$ are c-categories a smash pairing of $\mathscr{C}^{\prime}$ and $\mathscr{C}^{\prime \prime}$ to $\mathscr{C}$ is a functor $\#: \mathscr{C}^{\prime} \times \mathscr{C}^{\prime \prime} \rightarrow \mathscr{C}$ such that

(i) the functors $A^{\prime} \#-: \mathscr{C}^{\prime \prime} \rightarrow \mathscr{C}$ and $-\# A^{\prime \prime}: \mathscr{C}^{\prime} \rightarrow \mathscr{C}$ are c-functors for any $A^{\prime} \in \mathscr{C}^{\prime}, A^{\prime \prime} \in \mathscr{C}^{\prime \prime}$.

(ii) if $A^{\prime} \rightarrow B^{\prime}$ and $A^{\prime \prime} \rightarrow B^{\prime \prime}$ are cofibrations in $\mathscr{C}^{\prime}, \mathscr{C}^{\prime \prime}$ then

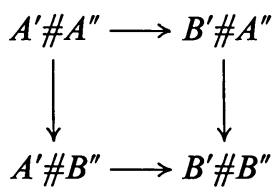

is a 2-cofibration.

If the categories in question are h-c-categories we shall further require

(iii) the functors $A^{\prime} \#-,-\# A^{\prime \prime}$ preserve homotopy.

The reader will readily supply examples; among them are the usual smash product for topological spaces and tensor products of modules.

We observe first that smash pairings of small c-categories or h-c-categories extend uniquely to their c-completions.

Proposition 14.1. If $\mathscr{C}_{0}, \mathscr{C}_{0}^{\prime}, \mathscr{C}_{0}^{\prime \prime}$ are small c-categories (h-c-categories) with c-completions $\mathscr{C}, \mathscr{C}^{\prime}, \mathscr{C}^{\prime \prime}$ and $\#_{0}: \mathscr{C}_{0}^{\prime} \times \mathscr{C}_{0}^{\prime \prime} \rightarrow \mathscr{C}_{0}$ is a smash pairing then $\#_{0}$ extends to a smash pairing \#: $\mathscr{C}^{\prime} \times \mathscr{C}^{\prime \prime} \rightarrow \mathscr{C}$ such that each of the functors $X^{\prime} \#-,-\# X^{\prime \prime}$ preserves c-colimits. This extension is unique up to canonical isomorphism.

The uniqueness will follow immediately from the preservation of c-colimits by the partial functors $X^{\prime} \#-,-\# X^{\prime \prime}$. The existence is provided by the Kan extension in the following manner.

If $X^{\prime} \in C^{\prime}$ we write $\left(\mathscr{C}_{0}^{\prime}, X^{\prime}\right)$ for the category of objects in $\mathscr{C}_{0}^{\prime}$ over $X^{\prime}$, i.e., of morphisms $a^{\prime}: A^{\prime} \rightarrow X^{\prime}$ with $A^{\prime} \in \mathscr{C}_{0}^{\prime}$. If also $X^{\prime \prime} \in \mathscr{C}^{\prime \prime}$ a functor $T:\left(\mathscr{C}_{0}^{\prime}, X^{\prime}\right)$ $\times\left(\mathscr{C}_{0}^{\prime \prime}, X^{\prime \prime}\right) \rightarrow \mathscr{C}$ is defined by $\left(a^{\prime}: A^{\prime} \rightarrow X^{\prime}, a^{\prime \prime}: A^{\prime \prime} \rightarrow X^{\prime \prime}\right) \rightarrow A^{\prime} \#_{0} A^{\prime \prime}$. But $X^{\prime}$ and $X^{\prime \prime}$ are colimits of c-systems, say $W^{\prime} \in c\left(\Lambda^{\prime}, \mathscr{C}_{0}^{\prime}\right)$ and $W^{\prime \prime} \in c\left(\Lambda^{\prime \prime}, \mathscr{C}_{0}^{\prime \prime}\right)$. These define 
cofinal functors $\Lambda^{\prime} \rightarrow\left(\mathscr{C}_{0}^{\prime}, X^{\prime}\right), \Lambda^{\prime \prime} \rightarrow\left(\mathscr{C}_{0}^{\prime \prime}, X^{\prime \prime}\right)$. Now for $\lambda^{\prime} \in \Lambda^{\prime}, \lambda^{\prime \prime} \rightarrow W_{\lambda}^{\prime} \#_{0} W_{\lambda^{\prime \prime}}^{\prime \prime}$ is again a c-system. We conclude that $T$ has a colimit, which may be computed as

$$
\begin{aligned}
\operatorname{colim} T & =\operatorname{colim}_{\lambda^{\prime}} \operatorname{colim}_{\lambda^{\prime \prime}} W_{\lambda^{\prime}}^{\prime} \#_{0} W_{\lambda^{\prime \prime}}^{\prime \prime} \\
& =\operatorname{colim}_{\lambda^{\prime \prime}} \operatorname{colim}_{\lambda^{\prime}} W_{\lambda^{\prime}}^{\prime} \#_{0} W_{\lambda^{\prime \prime}}^{\prime \prime}
\end{aligned}
$$

The properties of \#, defined by $X^{\prime} \# X^{\prime \prime}=\operatorname{colim} T$, follow routinely.

A smash pairing \# of h-c-categories defines, in virtue of (iii) a pairing of the corresponding homotopy categories which we again denote by \#. In the situation of 14.1 , if $\mathscr{C}_{0}, \mathscr{C}_{0}^{\prime}, \mathscr{C}_{0}^{\prime \prime}$ are connective h-c-categories then, \#: $\mathscr{C}^{\prime \square} \times \mathscr{C}^{\prime \prime} \square \rightarrow \mathscr{C}^{\square}$ extends $\#_{0}: \mathscr{C}_{0}^{\prime \square} \times \mathscr{C}_{0}^{\prime \prime \square} \rightarrow \mathscr{C}_{0}^{\square}$. In view of conditions (i) and (iii) we may apply 12.4 to get the following result.

Proposition 14.2. Functors $\mathscr{M}^{\prime}: \mathscr{C}^{\prime \square \text { op }} \times \mathscr{C}^{\square} \rightarrow \mathscr{C}^{\prime \prime \square}$ and $\mathscr{M}^{\prime \prime}: \mathscr{C}^{\prime \prime} \square \mathrm{op} \times \mathscr{C}^{\square} \rightarrow \mathscr{C}^{\prime \square}$ may be defined by the conditions that for $X^{\prime} \in \mathscr{C}^{\prime}\left(X^{\prime \prime} \in \mathscr{C}^{\prime \prime}\right)$ the functor $\mathscr{M}^{\prime}\left(X^{\prime},-\right)$ is adjoint to $X^{\prime} \#-: \mathscr{C}^{\prime \prime} \square \rightarrow \mathscr{C}^{\square},\left(\mathscr{M}^{\prime \prime}\left(X^{\prime \prime},-\right)\right.$ is adjoint to $\left.-\# X^{\prime \prime}: \mathscr{C}^{\prime} \rightarrow \rightarrow \mathscr{C}^{\square}\right)$.

If \#: $\mathscr{C} \times \mathscr{C} \rightarrow \mathscr{C}$ is a smash pairing which is monoidal, i.e., coherently associative, commutative and supplied with a unit (cf. [7], [4]), we shall call it a smash product in $\mathscr{C}$. Once more, examples abound. Since the topological one comes most readily to mind we shall always denote the unit of a smash product by $S^{0}$.

The Kan extension of a smash product in a small h-c-category remains monoidal and is thus a smash product in the c-completion. Moreover the pairing induced in the homotopy category is monoidal as well. In view of 14.2, we draw the following conclusion.

THEOREM 14.3. If $\#_{0}: \mathscr{C}_{0} \times \mathscr{C}_{0} \rightarrow \mathscr{C}_{0}$ is a smash product in the small connective h-c-category $\mathscr{C}_{0}$ then the Kan extension \#: $\mathscr{C} \times \mathscr{C} \rightarrow \mathscr{C}$ to the c-completion gives to $\mathscr{C} \square$ the structure of a monoidal closed category.

For the last notion, cf. once more [4].

15. Smash products and stable homotopy. If $\mathscr{C}$ is an h-c-category with a smash product we can always introduce, in many ways, a lifted suspension functor in $\mathscr{C}$. It then becomes natural to ask whether there is an induced smash product in the stabilization of $\mathscr{C}$ and consequently, when $\mathscr{C}$ is small, in its Boardman completion. If the answer is affirmative then the homotopy category of the Boardman completion becomes, by 14.3 , a monoidal closed category.

Unfortunately our understanding of this problem appears to be inadequate. Examples provided by chain complexes of modules over a commutative ring show that in some cases there is indeed a stable smash product. Starting on the other hand with the category of finite $\mathrm{CW}$-complexes it seems difficult or impossible to find a smash product in the stabilization. We have however no means of differentiating categories with from those without stable smash products nor, for that matter, any example illustrating conclusively the absence of such a product. 
It is however always possible to introduce a smash pairing of the stabilization to itself: what is in question is the commutativity and associativity of such a pairing. The question is further complicated by the fact that the pairing is not unique, being determined only up to a noncanonical isomorphism, and might thus be associative and commutative without being coherently so. Moreover it might well be the case that while the pairing itself lacks these properties the induced pairing on the homotopy category might be associative, commutative and even monoidal.

We must leave these questions unresolved and be for the moment contented with the following cursory account of the stable homotopy pairing and its adjoint "function-space" functors. We shall omit also a discussion of the relations between these several functors and the cofibration triangulations in the stable homotopy categories, pending a more complete investigation of the problems cited above.

If $\mathscr{C}$ is a h-c-category with a smash product \# having a unit $S^{0}$ we may construct (nonuniquely) a cone $S^{0} \rightarrow C S^{0}$ and denote its cofiber by $S^{1} . \mathscr{C}$ is then supplied with not one but two lifted suspension functors, $\Sigma^{\prime}=S^{1} \#-$ and $\Sigma^{\prime \prime}=-\# S^{1}$. While it is not strictly correct to say that $\Sigma^{\prime}$ and $\Sigma^{\prime \prime}$ commute-the two compositions are, rather, connected by the associativity isomorphism of \#-the coherency of the associativity of \# makes it possible to treat them as though this were the case, and we shall abbreviate our argument by supposing it to be so.

As discussed in $\S 13$, then, $\Sigma^{\prime}$ and $\Sigma^{\prime \prime}$ give rise to functors $\Sigma^{\prime}: \operatorname{Stab}_{\Sigma^{\prime \prime}} \mathscr{C} \rightarrow \operatorname{Stab}_{\Sigma^{\prime \prime}} \mathscr{C}$ and $\Sigma^{\prime \prime}: \operatorname{Stab}_{\Sigma^{\prime}} \mathscr{C} \rightarrow \operatorname{Stab}_{\Sigma^{\prime}} \mathscr{C}$ and there are, further, stable functors

$$
\operatorname{Stab}_{\Sigma^{\prime}} \mathscr{C} \stackrel{U^{\prime}}{\longrightarrow} \operatorname{Stab}_{\Sigma^{\prime}, \Sigma^{\prime \prime}} \mathscr{C} \stackrel{U^{\prime \prime}}{\longrightarrow} \operatorname{Stab}_{\Sigma^{\prime \prime}} \mathscr{C}
$$

into the double stabilization.

Of course the commutativity isomorphism of \# makes $\Sigma^{\prime}$ and $\Sigma^{\prime \prime}$ isomorphic on $\mathscr{C}$. But it is to be noted that this isomorphism is not necessarily stable with respect to either suspension and thus need not give rise to an isomorphism in any of the stable categories.

It does however permit us to draw the following conclusion:

The categories $\operatorname{Stab}_{\Sigma^{\prime}} \mathscr{C}, \mathrm{Stab}_{\Sigma^{\prime \prime}} \mathscr{C}$ are weakly stable with respect to $\Sigma^{\prime \prime}, \Sigma^{\prime}$. Thus $U^{\prime}, U^{\prime \prime}$ are equivalences of categories.

For if $X \in \mathscr{C}$ the isomorphism $\Sigma^{\prime} X \approx \Sigma^{\prime \prime} X$ gives in $\operatorname{Stab}_{\Sigma^{\prime}} \mathscr{C}$, for any $n, \Sigma^{\prime n+1} X$ $\approx \Sigma^{\prime \prime} \Sigma^{\prime n} X$. The second assertion follows from the first via 13.1.

Now the smash product in $\mathscr{C}$ gives us, by twice applying the universal property of the stabilization, a smash pairing \#: $\operatorname{Stab}_{\Sigma^{\prime}} \mathscr{C} \times \operatorname{Stab}_{\Sigma^{\prime \prime}} \mathscr{C} \rightarrow \operatorname{Stab}_{\Sigma^{\prime}, \Sigma^{\prime \prime}} \mathscr{C}$, using the evident relation $\Sigma^{\prime} X^{\prime} \# \Sigma^{\prime \prime} X^{\prime \prime}=\Sigma^{\prime} \Sigma^{\prime \prime}\left(X^{\prime} \# X^{\prime \prime}\right)$. If $\bar{U}^{\prime}, \bar{U}^{\prime \prime}$ are inverses up to isomorphism of $U^{\prime}, U^{\prime \prime}$ then

$$
\bar{U}^{\prime} \circ \# \circ\left(1 \times \bar{U}^{\prime \prime} U^{\prime}\right), \quad \bar{U}^{\prime \prime} \circ \# \circ\left(\bar{U}^{\prime} U^{\prime \prime} \times 1\right), \quad \# \circ\left(\bar{U}^{\prime} \times \bar{U}^{\prime \prime}\right)
$$

are smash pairings in $\operatorname{Stab}_{\Sigma^{\prime}} \mathscr{C}, \operatorname{Stab}_{\Sigma^{\prime \prime}} \mathscr{C}, \operatorname{Stab}_{\Sigma^{\prime}, \Sigma^{\prime \prime}} \mathscr{C}$.

Let us suppose that $\mathscr{C}$ is a small category. Then 14.1 provides us with a canonical smash pairing \#: $\mathscr{B}_{\Sigma^{\prime}} \mathscr{C} \times \mathscr{B}_{\Sigma^{\prime \prime}} \mathscr{C} \rightarrow \mathscr{B}_{\Sigma^{\prime}, \Sigma^{\prime \prime}} \mathscr{C}$, where $\mathscr{B}_{\Sigma^{\prime}, \Sigma^{\prime \prime}} \mathscr{C}$ is the c-completion of 
$\operatorname{Stab}_{\Sigma^{\prime}, \Sigma^{\prime \prime}} \mathscr{C}$. Proposition 14.2 then applies and yields functors

$$
\begin{aligned}
& \mathscr{M}^{\prime}:\left(\mathscr{B}_{\Sigma^{\prime}} \mathscr{C}\right)^{\square \mathrm{op}} \times\left(\mathscr{B}_{\Sigma^{\prime}, \Sigma^{\prime \prime}} \mathscr{C}\right)^{\square} \rightarrow\left(\mathscr{B}_{\Sigma^{\prime \prime}} \mathscr{C}\right)^{\square}, \\
& \mathscr{M}^{\prime \prime}:\left(\mathscr{B}_{\Sigma^{\prime \prime}} \mathscr{C}\right)^{\square \mathrm{op}} \times\left(\mathscr{B}_{\Sigma^{\prime}, \Sigma^{\prime \prime}} \mathscr{C}\right)^{\square} \rightarrow\left(\mathscr{B}_{\Sigma^{\prime}} \mathscr{C}\right)^{\square}
\end{aligned}
$$

adjoint to the induced pairing on the homotopy categories. Since the three categories involved are equivalent, these may be interpreted as "function-space" functors in any one of them.

\section{REFERENCES}

1. J. M. Boardman, Stable homotopy theory, University of Warwick, Warwick, 1966.

2. E. H. Brown, Jr., Cohomology theories, Ann. of Math. (2) 75 (1962), 467-484. MR 25 \#1551.

3. A. Dold, Halbexakte Homotopiefunktoren, Lecture Notes in Math. no. 12, SpringerVerlag, New York, 1966. MR 33 \#6622.

4. S. Eilenberg and G. M. Kelly, Closed categories, Proc. Conf. Categorical Algebra (La Jolla, Calif., 1965), Springer-Verlag, New York, 1966, pp. 421-562. MR 37 \#1432.

5. P. J. Freyd, Stable homotopy, Proc. Conf. Categorical Algebra (La Jolla, Calif., 1965), Springer-Verlag, New York, 1966, pp. 121-172. MR 35 \#2280.

6. A. Heller, Stable homotopy categories, Bull. Amer. Math. Soc. 74 (1968), 28-63. MR 36 \#7137.

7. S. MacLane, Natural associativity and commutativity, Rice Univ. Studies 49 (1963), no. 4 , 28-46. MR 30 \#1160.

8. D. Puppe, Stabile Homotopietheorie. I, Math. Ann. 169 (1967), 243-274. MR 35 \#2281.

City University OF New YoRK,

NeW YorK, NEW YoRK 